\title{
Improved Inference for the Signal Significance
}

\author{
Igor Volobouev $^{*} \quad$ A. Alexandre Trindade, ${ }^{\dagger}$
}

August 9, 2021

\begin{abstract}
We study the properties of several likelihood-based statistics commonly used in testing for the presence of a known signal under a mixture model with known background, but unknown signal fraction. Under the null hypothesis of no signal, all statistics follow a standard normal distribution in large samples, but substantial deviations can occur at low sample sizes. Approximations for respective $p$-values are derived to various orders of accuracy using the methodology of Edgeworth expansions. Adherence to normality is studied, and the magnitude of deviations is quantified according to resulting $p$-value inflation or deflation. We find that approximations to third-order accuracy are generally sufficient to guarantee $p$-values with nominal false positive error rates in the $5 \sigma$ range $\left(p\right.$-value $\left.=2.87 \times 10^{-7}\right)$ for the classic Wald, score, and likelihood ratio (LR) statistics at relatively low samples. Not only does LR have better adherence to normality, but it also consistently outperforms all other statistics in terms of false negative error rates. The reasons for this are shown to be connected with high-order cumulant behavior gleaned from fourth order Edgeworth expansions. Finally, a conservative procedure is suggested for making finite sample adjustments while accounting for the look elsewhere effect with the theory of random fields (a.k.a. the Gross-Vitells method).
\end{abstract}

\section{Introduction}

In particle physics, a typical search for a new particle or resonance consists in studying invariant masses of decay products and identifying a narrow, localized signal spike on top of a slowly varying background. If the main purpose of the study is determination of signal existence, the mass of the particle enters the analysis as the model nuisance parameter. When it can be expected that the natural width of the particle under search is small, one can usually assume that the shape of the hypothesized signal is Gaussian and that its width is determined by the detector resolution. On the other hand, if the expected width is comparable to or substantially larger than the resolution, it has to be treated as another nuisance parameter in the search.

Reliable determination of the frequentist statistical significance of signal evidence, i.e., the $p$ value, is crucial for substantiating any claim of discovery. The $p$-value is derived by estimating how often the statistical fluctuations in the background alone can produce an apparent signal excess similar to or larger than that observed in the data. However, the parameters of the signal, such as its location and width, are no longer identifiable in a model which represents pure background. As the Wilks' theorem [18] does not hold in such situations, the classical nuisance parameter treatment based on profile likelihood no longer results in a simple asymptotic behavior of the $p$-value [5].

In particle physics, the problem of proper accounting for the false positive signal detection over a wide search area is referred to as the look elsewhere effect (LEE) [11]. While this effect and

* Department of Physics \& Astronomy, Texas Tech University

${ }^{\dagger}$ Department of Mathematics \& Statistics, Texas Tech University 
its associated "trial factor" (i.e., the increase in the $p$-value in comparison with the case of fixed nuisances) can be estimated numerically, reliable direct determination of the $p$-value by computer simulations is often prohibitively expensive in terms of required CPU time. Fortunately, an accurate asymptotic treatment of this problem is possible with the aid of techniques developed within the theory of random fields $[1,2]$. The statistic used to test for signal presence is associated with a random field in the space formed by nuisance parameters. The probability that the field maximum exceeds some level depends on the postulated geometry of the parameter space and on the field covariance function. It turns out that, if a sufficiently high level is chosen, the asymptotic behavior of the $p$-value is determined by a few coefficients only ${ }^{1}$, and full knowledge of the covariance function is not necessary. These coefficients can be evaluated numerically at a much reduced computational cost in comparison with the brute-force $p$-value determination. In application to particle physics searches, this approach has became known as the Gross-Vitells method [3,7,17].

Determination of the LEE trial factor by the theory of random fields relies upon the crucial assumption that the pointwise distributions of these fields are known. The relevant theory is developed for centered Gaussian fields with unit variance and a few related fields, such as $\chi^{2}$. However, for finite samples, departures of the signal testing statistic from normality can be substantial. To ensure applicability of the LEE theory, we must assess how well its assumptions are satisfied and constrain deviations from these assumptions. With this purpose in mind, we investigate final sample effects for a number of statistics that can be used to claim signal discovery. The specific problem we wish to address is accurate testing for the presence of a signal fraction, $\alpha$, in the context of a model involving an additive combination of signal and background distributions. In what follows, it will be assumed that both the signal and background are described by known probability density functions, $s(x)$ and $b(x)$ respectively, for some univariate or multivariate $x$, and that the data are fitted by maximum likelihood to the (mixture) model density:

$$
p(x \mid \alpha)=\alpha s(x)+(1-\alpha) b(x) .
$$

Here, $\alpha$ is the parameter of interest, to be inferred from available data. For searches with unknown signal location and/or width, this model corresponds to a single point in the nuisance parameter space. If the observed sample points $x_{1}, \ldots, x_{n}$ consist of independent and identically distributed realizations from model (1), the log-likelihood is

$$
\ell(\alpha)=\sum_{i=1}^{n} \log p\left(x_{i} \mid \alpha\right) .
$$

The maximum likelihood estimator (MLE) $\hat{\alpha}$ is obtained by maximizing $\ell(\alpha)$ over all $\alpha \in \mathbb{R}$. It is easily seen that $\ell(\alpha)$ is strictly concave under continuous background and signal models, and thus $\hat{\alpha}$ is straightforward to locate numerically. The overall goal is to produce accurate tests of

$$
\mathcal{H}_{0}: \alpha=0 \quad \text { vs. } \quad \mathcal{H}_{1}: \alpha>0
$$

In the statistics literature, (1) is known as a mixture model [13]. Asymptotically (i.e., for $n \rightarrow$ $\infty), \hat{\alpha}$ is consistent, normal, and efficient under mild regularity conditions ${ }^{2}$. In practice, however,

\footnotetext{
${ }^{1}$ These are the coefficients that enter the asymptotic expansion of the Euler characteristic of the field excursion set.

${ }^{2}$ It should be pointed out that in this study we do not impose the $\alpha \in[0,1]$ constraint. In practice, this constraint would not lead to a noticeable improvement in the result iterpretability. At the same time, it would introduce an unnecessary complication in various derivations by breaking the likelihood regularity conditions at the boundaries and by adding a point mass at 0 to the $\hat{\alpha}$ distribution.
} 
we must maintain confidence in the statements made for finite $n$. Deviations from normality can be assessed analytically through so-called "higher-order" statistical inference techniques [15]. In this manuscript, we develop higher-order approximations for model (1) with the purpose to both provide such an assessment and improve frequentist coverage of the relevant tests. As outlined in section 7 , adjustments made to the local significance of the test statistic can also be translated into a subsequent conservative estimate of the global $p$-value.

If $b(x)$ has nuisance parameters, the likelihood must be profiled over these parameters. Although we do not specifically tackle it here, application of the techniques detailed in [15] is also possible in this situation, leading to suitably modified versions of the asymptotic expansions we derive in the current paper ${ }^{3}$. Another complication we do not address here is the possibility for the sample size $N$ to be random, since in a typical particle physics experiment $N$ is Poisson-distributed. However, the analysis we present here is conditional on a fixed value of $N=n$ when forming (2).

The remainder of the paper is organized as follows. Section 2 introduces the statistics that will be used to test (3), and provides an overview of the higher-order inference techniques to be used for approximating the resulting $p$-values, that will be presented in detail in section 3 . After describing an example that poignantly illustrates the need for $p$-value adjustment in section 4 , we confirm the validity of the proposed approximations via some simulation experiments in section 5 . Finally, section 6 undertakes a study of type I and type II errors. We end the paper with a discussion.

\section{Higher-Order Inference Techniques}

To set up the notation for what follows, we denote by $\ell_{i}(\alpha)=\partial^{i} \ell / \partial \alpha^{i}$ the $i$-th derivative of $\ell(\alpha)$. Now define $J(\alpha)=-\ell_{2}(\alpha)$, and the expected information number as $I(\alpha)=\mathbb{E} J(\alpha)$. Also implicit in the notation is the argument at which a particular derivative is calculated, e.g., $J(\hat{\alpha})=-\left.\ell_{2}(\alpha)\right|_{\alpha=\hat{\alpha}}$, which is the usual definition of the observed information number. We assume that the usual regularity conditions for consistency and asymptotic normality of $\hat{\alpha}$ are satisfied, e.g., [15, ch. 3]. Thus, we place no restrictions on the parameter space, so that $\alpha \in \mathbb{R}$.

Parametric statistical inference then seeks a statistic $T \equiv T(\boldsymbol{x})$, a function of the data vector $\boldsymbol{x}$, which is used to formulate a rejection rule for the null hypothesis $\mathcal{H}_{0}$, thereby providing, in some quantifiable sense, an optimal testing procedure (see, e.g., [10] for a detailed exposition relating to the notion of a uniformly most powerful, or UMP, test). The three classical test statistics most commonly used in this context are the Likelihood Ratio (LR), Wald (two versions), and Score, defined in Table 1. It is well known that in lack of a UMP test, these statistics, and LR in particular, are generally near-optimal [16].

Table 1: Definition of the most common versions of the Likelihood Ratio, Wald, and Score statistics for testing the hypothesis in (3).

\begin{tabular}{llc}
\hline Method & Statistic & Value \\
\hline Likelihood Ratio & $T_{\mathrm{LR}}$ & $2[\ell(\hat{\alpha})-\ell(0)]$ \\
Wald (Expected) & $T_{\mathrm{W}}$ & $\hat{\alpha}^{2} I(0)$ \\
Wald (Observed) & $T_{\mathrm{W} 2}$ & $\hat{\alpha}^{2} J(\hat{\alpha})$ \\
Score & $T_{\mathrm{S}}$ & $\ell_{1}(0)^{2} / I(0)$ \\
Wald-type 3 & $T_{\mathrm{W} 3}$ & $\hat{\alpha}^{2} / \sigma_{3}^{2}$ \\
Wald-type 4 & $T_{\mathrm{W} 4}$ & $\hat{\alpha}^{2} / \sigma_{4}^{2}$ \\
\hline
\end{tabular}

\footnotetext{
${ }^{3}$ However, the required calculations are substantially more challenging.
} 
Since the asymptotic (large sample) variance of the MLE $\hat{\alpha}$ is $\sigma_{\hat{\alpha}}^{2}=I\left(\alpha_{0}\right)^{-1}$, where $\alpha_{0}$ denotes the true or hypothesized value of $\alpha$, the Wald statistics can use any of the consistent estimators $I\left(\alpha_{0}\right)^{-1}, I(\hat{\alpha})^{-1}, J\left(\alpha_{0}\right)^{-1}$, or $J(\hat{\alpha})^{-1}$, when standardizing it (the expected and observed versions listed in Table 1 being the ones in most common usage). The two Wald-type statistics appearing at the bottom of Table 1 , are variants of $T_{\mathrm{W} 2}$ that use a shortcut for computing $\sigma_{\hat{\alpha}}^{2}$ so as to circumvent the need to explicitly differentiate $\ell(\alpha)$. Specifically, an estimate of the $\hat{\alpha}$ uncertainty is obtained by locating the two roots of $\ell(\hat{\alpha})-\ell(\alpha)=1 / 2$; this technique enjoying wide acceptance in particle physics after its popularization by $[6,8]$. Letting $\alpha_{\min }<\alpha_{\max }$ denote the two solutions in question, define $\sigma^{-}=\hat{\alpha}-\alpha_{\min }$ and $\sigma^{+}=\alpha_{\max }-\hat{\alpha}$. Two additional approaches for estimating $\sigma_{\hat{\alpha}}$ are then $\sigma_{3}=\left(\sigma^{+}+\sigma^{-}\right) / 2$, and $\sigma_{4}=\sigma^{-}$.

Under $\mathcal{H}_{0}$, the LR, Wald, and Score statistics are asymptotically distributed as $\chi_{1}^{2}$, to first order $^{4}$. We can write this concisely as $T \dot{\sim} \chi_{1}^{2}$, valid to $O\left(n^{-1 / 2}\right)$. The statement that $T \dot{\sim} \chi_{1}^{2}$ to $k$-th order, or $O\left(n^{-k / 2}\right)$, informally means that for finite $n$ a corrected quantity $\left(1+O\left(n^{-k / 2}\right)\right) T+$ $O\left(n^{-k / 2}\right)$ can be found which is distributed as $\chi_{1}^{2}$, for $k=1,2,3, \ldots$. In many situations, it is possible to construct higher-order approximations $(k \geq 2)$ explicitly. These are arranged in powers of $n^{-1 / 2}$, and give us control over the differences between the finite $n$ distribution of a statistic and its limiting behavior.

For testing the one-sided alternative $\mathcal{H}_{1}$, the signed version of any of the statistics in Table 1 (say $T$ ) can be used, by defining

$$
R=\operatorname{sgn}(\hat{\alpha}) \sqrt{T} \text {. }
$$

In this case, and under $\mathcal{H}_{0}, R$ is asymptotically distributed as a standard normal, $\mathcal{N}(0,1)$, to first order, whence the corresponding $p$-value is $P(\mathcal{N}(0,1)>r)$, where $r=\operatorname{sgn}(\hat{\alpha}) \sqrt{t}$ and $t$ is the observed value of $T$ calculated from the sample on hand. In the ensuing discussion we reserve the symbols $\Phi(\cdot)$ and $\phi(\cdot)$ for the cumulative distribution and probability density functions, respectively, of a $\mathcal{N}(0,1)$ distribution.

Tools for developing higher-order asymptotic theory include Taylor series expansions of $\ell(\cdot)$ near $\alpha=0$, joint cumulants for the derivatives of $\ell(\cdot)$, and Edgeworth approximations to distributions. To any finite order, relationships between cumulants and moments can be determined routinely via a symbolic algebra system (starting from the Taylor expansion of the cumulant generating function). To obtain Edgeworth approximations to the probabilities of a given statistic, its cumulants are expressed in terms of the joint cumulants of the log-likelihood derivatives. The essence of these approximations (for a random variable $Z$ whose distribution is close to standard normal) is to construct an approximate density with the Gram-Charlier expansion

$$
f(z)=\phi(z)\left(1+\sum_{j=1}^{\infty} \beta_{j} H_{j}(z)\right)
$$

whereby the coefficients $\beta_{j}$ are chosen to match the cumulants $\kappa_{j}$ of the approximated distribution. The corresponding cumulative distribution function (CDF) for $f(z), F(z)=\int_{-\infty}^{z} f(x) d x$, is easily found using the following property of the Hermite polynomials ${ }^{5}$ in $(5), \int_{-\infty}^{z} H_{j}(x) e^{-x^{2} / 2} d x=$ $-H_{j-1}(z) e^{-z^{2} / 2}$, whence

$$
F(z)=\Phi(z)-\phi(z) \sum_{j=1}^{\infty} \beta_{j} H_{j-1}(z)=1-S(z),
$$

\footnotetext{
${ }^{4}$ This fact remains true for Wald and Score if any of the following versions of "information number" are used in the definition of the statistic: $I(0), I(\hat{\alpha}), J(0)$, or $J(\hat{\alpha})$.

${ }^{5}$ For example: $H_{2}(z)=z^{2}-1, H_{3}(z)=z^{3}-3 z$, and $H_{5}(z)=z^{5}-10 z^{3}+15 z$.
} 
where $S(z)$ is the so-called survival function (or right-tail probability). The final Edgeworth expansion is now obtained from (6) by collecting terms in powers of $n^{-1 / 2}$ :

$$
\begin{aligned}
F(z)=\Phi(z)-\phi(z)\left[\kappa_{1}+\frac{1}{6} \kappa_{3} H_{2}(z)+\right. & \frac{1}{2}\left(\kappa_{1}^{2}+\kappa_{2}-1\right) z \\
& \left.+\left(\frac{1}{6} \kappa_{1} \kappa_{3}+\frac{1}{24} \kappa_{4}\right) H_{3}(z)+\frac{1}{72} \kappa_{3}^{2} H_{5}(z)+O\left(n^{-3 / 2}\right)\right] .
\end{aligned}
$$

This expansion assumes the following (typical) cumulant behavior for $Z$ : $\kappa_{1}=O\left(n^{-1 / 2}\right), \kappa_{2}=$ $1+O\left(n^{-1}\right)$, and $\kappa_{m}=O\left(n^{-(m-2) / 2}\right)$ for all $m>2$. It then follows that the first four cumulants of $F(z)$ are $\kappa_{1}, \kappa_{2}, \kappa_{3}+O\left(n^{-3 / 2}\right)$, and $\kappa_{4}+O\left(n^{-3 / 2}\right)$, with all subsequent cumulants being $O\left(n^{-3 / 2}\right)$ or smaller. With the exception of $R_{\mathrm{W} 4}$, all signed versions of the statistics in Table 1 exhibit this cumulant behavior. The appropriate version of (7) for the case in which $\kappa_{2}=1+O\left(n^{-1 / 2}\right)$ is given in Appendix B.

The fundamental strategies in seeking improved inference in the finite $n$ situation fall into the following categories.

Strategy 1. More accurate approximations to tail probabilities of $R$ in the form:

$$
P(R \leq r)=\Phi(r)+\text { correction }+ \text { error }
$$

where the "correction" is a function of both $n$ and $r$, and the "error" is $O\left(n^{-1}\right)$ or smaller. These are accomplished primarily through Edgeworth expansions. Throughout the paper we refer to the resulting tail probabilities as Edgeworth "approximations" or "predictions".

Strategy 2. Modifications to $R$, so that $R \mapsto \tilde{R}$, with $\tilde{R}$ more closely following a $\mathcal{N}(0,1)$ :

$$
P(\tilde{R} \leq r)=\Phi(r)+\text { error }
$$

and the "error" is $O\left(n^{-1}\right)$ or smaller. The challenge in these modifications is to correct towards normality, while still retaining the essence (and near-optimal properties) of $R$.

These strategies apply also to $T=R^{2}$; the only change being that $\Phi(\cdot) \mapsto 2 \Phi(\cdot)-1$, corresponding to a $\chi_{1}^{2}$ tail probability. Approaches under both strategies were gradually developed over the past century, with some of the most important contributions emerging from the pioneering work of [4]. A more recent and updated treatment of the methodology is given by [15]. In the next section we detail the explicit calculations involved.

\section{Approximated $p$-Values and Normalizing Transformations}

To concisely compute the approximate $p$-values for the statistics under study, we define two versions of the expectation operator: $\mathbb{E}$ and $\mathbb{E}_{s}$ will denote expectation under $\mathcal{H}_{0}$ and under the signal, respectively:

$$
\mathbb{E}[q]:=\int q(x) b(x) d x, \quad \mathbb{E}_{s}[q]:=\int q(x) s(x) d x .
$$


Additionally, with the notation $V_{i}:=\mathbb{E}\left[\ell_{i}(0)\right]$, we find that the following (dimensionless and location-scale invariant) quantities play a key role in the expressions below:

$$
\begin{aligned}
\gamma & =\frac{V_{3}}{2\left(-V_{2}\right)^{3 / 2}}=\frac{\mathbb{E}_{s}\left[\frac{s^{2}}{b^{2}}\right]-3 \mathbb{E}_{s}\left[\frac{s}{b}\right]+2}{\left(\mathbb{E}_{s}\left[\frac{s}{b}\right]-1\right)^{3 / 2}}, \\
\rho & =-\frac{V_{4}}{6 V_{2}^{2}}=\frac{\mathbb{E}_{s}\left[\frac{s^{3}}{b^{3}}\right]-4 \mathbb{E}_{s}\left[\frac{s^{2}}{b^{2}}\right]+6 \mathbb{E}_{s}\left[\frac{s}{b}\right]-3}{\left(\mathbb{E}_{s}\left[\frac{s}{b}\right]-1\right)^{2}} .
\end{aligned}
$$

We now give, under Strategy 1, explicit higher-order expansions for the signed versions of the statistics from Table 1. The bulk of the work involves computing joint cumulants for the derivatives of $\ell(\cdot)$; these forming the basis for approximating the cumulants that are then substituted into (7). Specifically, and following [15, ch. 5], in what follows we denote by $n \nu_{i j k l}$ the $(i, j, k, l)$-th cumulant ${ }^{6}$ of the first four derivatives of $\ell(\alpha)$ evaluated at $\alpha=0,\left\{\ell_{1}(0), \ldots, \ell_{4}(0)\right\}$. The calculation of these cumulants is detailed in Appendix A. Proposed approaches to improving inference under the guise of Strategy 2 are also briefly discussed.

\subsection{Strategy 1: approximations to $p$-values}

For testing $\mathcal{H}_{0}$ vs. $\mathcal{H}_{1}$ under the paradigm of Strategy 1, we compute higher-order Edgeworth expansions for the tail probabilities of signed versions of the statistics in Table 1. First, starting from the approximated $R_{\mathrm{LR}}$ cumulants given in [15, sec. 5.4], straightforward calculations give the corresponding approximated cumulants for model (2) listed in the first row of Table 2. Substituting these expressions into (7) gives immediately,

$$
P\left(R_{\mathrm{LR}} \leq z\right)=\Phi(z)-\phi(z)\left[\left(-\frac{\gamma}{6}\right) n^{-1 / 2}+\left(\frac{\left(3 \rho-2 \gamma^{2}\right) z}{12}\right) n^{-1}+O\left(n^{-3 / 2}\right)\right] .
$$

Next, we give analogous results for the expected and observed versions of Wald. This entails first approximating the appropriate cumulants, expressions for which are given in [15, sec. 5.3] to third order accuracy for $R_{\mathrm{W}}$. The first four cumulants appear to be correctly stated except for the $2 \mathrm{nd}$, the correct version of which should be:

$$
n\left[\hat{\kappa}_{2}\left(R_{\mathrm{W}}\right)-1\right]=\left(2 \nu_{21}+3 \nu_{101}+3 \nu_{02}+\nu_{0001}\right) \nu_{2}^{-2}+\left(\nu_{001} \nu_{3}+\frac{7 \nu_{001}^{2}}{2}+5 \nu_{11}^{2}+11 \nu_{11} \nu_{001}\right) \nu_{2}^{-3} .
$$

Straightforward calculations then give the values in the second row of Table 2. Substitution of these into (7) gives eventually

$$
\begin{aligned}
P\left(R_{\mathrm{W}} \leq z\right)=\Phi(z)-\phi(z) & {\left[\left(\frac{\gamma H_{2}(z)}{6}\right) n^{-1 / 2}+\right.} \\
& \left.\left(\frac{\left(\rho-\gamma^{2}-1\right) z}{2}+\frac{(\rho-3) H_{3}(z)}{24}+\frac{\gamma^{2} H_{5}(z)}{72}\right) n^{-1}+O\left(n^{-3 / 2}\right)\right] .
\end{aligned}
$$

For the observed version of Wald, [15, sec. 5.3] carries only second order accuracy in the approximations to the cumulants of $R_{\mathrm{W} 2}$. Working from first principles (using Taylor series expansions of $\ell(\cdot)$ with an appropriate number of terms) and following the general procedure outlined in $[15$, ch. 5$]$

\footnotetext{
${ }^{6}$ An abbreviated notation will be adhered to by omitting trailing zeros, e.g., $\nu_{1020} \equiv \nu_{102}$.
} 
for Edgeworth expansions, we obtain the following third-order accurate expressions for the first four cumulants of $R_{\mathrm{W} 2}$ :

$$
\begin{gathered}
\hat{\kappa}_{1}\left(R_{\mathrm{W} 2}\right)=\frac{\nu_{11}}{2 \nu_{2}^{3 / 2} \sqrt{n}}, \quad \hat{\kappa}_{3}\left(R_{\mathrm{W} 2}\right)=-\frac{\nu_{001}}{\nu_{2}^{3 / 2} \sqrt{n}}, \\
n\left[\hat{\kappa}_{2}\left(R_{\mathrm{W} 2}\right)-1\right]=\left(\nu_{21}+\nu_{02}-\frac{\nu_{0001}}{2}\right) \nu_{2}^{-2}+\left(\frac{7 \nu_{11}^{2}}{4}-\frac{3 \nu_{001}^{2}}{4}\right) \nu_{2}^{-3},
\end{gathered}
$$

and

$$
n \hat{\kappa}_{4}\left(R_{\mathrm{W} 2}\right)=\left(\nu_{4}+6 \nu_{21}+3 \nu_{02}-2 \nu_{0001}\right) \nu_{2}^{-2}+\left(6 \nu_{11} \nu_{3}+18 \nu_{11}^{2}-3 \nu_{001}^{2}\right) \nu_{2}^{-3} .
$$

The conversion of these expressions in terms of our log-likelihood function (2) is listed in Table 2. Substitution of these values into (7) gives

$$
\begin{aligned}
P\left(R_{\mathrm{W} 2} \leq z\right)=\Phi(z)-\phi(z) & {\left[-\left(\frac{3 \gamma+2 \gamma H_{2}(z)}{6}\right) n^{-1 / 2}+\right.} \\
& \left.\left(\frac{\left(3 \rho-\gamma^{2}\right) z}{2}+\frac{\left(5 \rho+2 \gamma^{2}\right) H_{3}(z)}{12}+\frac{\gamma^{2} H_{5}(z)}{18}\right) n^{-1}+O\left(n^{-3 / 2}\right)\right] .
\end{aligned}
$$

Following the same procedure as for $R_{\mathrm{W} 2}$, we obtain analogous third-order accurate expressions for the first four cumulants of $R_{\mathrm{S}}$ :

$$
\hat{\kappa}_{1}\left(R_{\mathrm{S}}\right)=0, \quad \hat{\kappa}_{2}\left(R_{\mathrm{S}}\right)=1, \quad \hat{\kappa}_{3}\left(R_{\mathrm{S}}\right)=\frac{\nu_{3}}{\nu_{2}^{3 / 2} \sqrt{n}}, \quad \hat{\kappa}_{4}\left(R_{\mathrm{S}}\right)=\frac{\nu_{4}}{n \nu_{2}^{2}}
$$

The conversion of these expressions into our log-likelihood function appears in Table 2. Substitution of these $R_{\mathrm{S}}$ cumulants into (7) gives

$$
P\left(R_{\mathrm{S}} \leq z\right)=\Phi(z)-\phi(z)\left[\left(\frac{\gamma H_{2}(z)}{6}\right) n^{-1 / 2}+\left(\frac{(\rho-3) H_{3}(z)}{24}+\frac{\gamma^{2} H_{5}(z)}{72}\right) n^{-1}+O\left(n^{-3 / 2}\right)\right] .
$$

Finally, Table 2 also includes $O\left(n^{-3 / 2}\right)$ cumulants for $R_{\mathrm{W} 3}$ and $R_{\mathrm{W} 4}$, obtained by similar calculations. The tail probabilities for these statistics are given in Appendix B.

Table 2: Approximations to the first four cumulants of the signed versions of the statistics in Table 1, in the context of log-likelihood expression (2). The error in these approximations is $O\left(n^{-3 / 2}\right)$.

\begin{tabular}{lcccc}
\hline Statistic & $\hat{\kappa}_{1}$ & $\hat{\kappa}_{2}$ & $\hat{\kappa}_{3}$ & $\hat{\kappa}_{4}$ \\
\hline$R_{\mathrm{LR}}$ & $-\frac{\gamma}{6 \sqrt{n}}$ & $1+\frac{18 \rho-13 \gamma^{2}}{36 n}$ & 0 & 0 \\
$R_{\mathrm{W}}$ & 0 & $1+\frac{\rho-\gamma^{2}-1}{n}$ & $\frac{\gamma}{\sqrt{n}}$ & $\frac{\rho-3}{n}$ \\
$R_{\mathrm{W} 2}$ & $-\frac{\gamma}{2 \sqrt{n}}$ & $1+\frac{12 \rho-5 \gamma^{2}}{4 n}$ & $-\frac{2 \gamma}{\sqrt{n}}$ & $\frac{10 \rho}{n}$ \\
$R_{\mathrm{S}}$ & 0 & 1 & $\frac{\gamma}{\sqrt{n}}$ & $\frac{\rho-3}{n}$ \\
$R_{\mathrm{W} 3}$ & $-\frac{\gamma}{2 \sqrt{n}}$ & $1+\frac{126 \rho-65 \gamma^{2}}{36 n}$ & $-\frac{2 \gamma}{\sqrt{n}}$ & $\frac{10 \rho}{n}$ \\
$R_{\mathrm{W} 4}$ & $-\frac{\gamma}{2 \sqrt{n}}-\frac{3 \rho-\gamma^{2}}{3 n}$ & $1+\frac{2 \gamma}{3 \sqrt{n}}+\frac{126 \rho-53 \gamma^{2}}{36 n}$ & $-\frac{2 \gamma}{\sqrt{n}}-\frac{4 \rho-\gamma^{2}}{n}$ & $\frac{10 \rho}{n}$ \\
\hline
\end{tabular}




\subsection{Strategy 2: normalizing transformations}

A general transformation for "normalizing" the distribution of a statistic $R$ that is already approximately normal, is

$$
\tilde{R}(r)=\Phi^{-1}(P(R \leq r)),
$$

where $r$ denotes the observed value of $R$ computed from the sample at hand. The key idea is that by invoking an accurate (higher-order) approximation to the cumulative probabilities that comprise the argument of the standard normal quantile function $\Phi^{-1}(\cdot)$, the probability integral transform will then ensure better compliance with a Gaussian distribution. For typical expansions considered in this study,

$$
P(R \leq r)=\Phi(r)-\phi(r)\left[\frac{a(r)}{\sqrt{n}}+\frac{b(r)}{n}+O\left(n^{-3 / 2}\right)\right],
$$

numerically stable evaluation of (14) to $O\left(n^{-3 / 2}\right)$ can be performed at large $r$ by calculating

$$
\tilde{R}(r)=S_{\Phi}^{-1}\left(S_{\Phi}(r)+\phi(r)\left[\frac{a(r)}{\sqrt{n}}+\frac{b(r)}{n}\right]\right)
$$

where $S_{\Phi}(z) \equiv 1-\Phi(z)$ is the survival function of a $\mathcal{N}(0,1)$. This technique, in essence a combination of Strategies 1 and 2, would therefore be immediately applicable to any of the statistics from Table 2.

On the other hand, [15, ch. 7] discusses at length a classical modification to the signed LR statistic

$$
R_{\mathrm{LR}}(\alpha)=\operatorname{sgn}(\hat{\alpha}) \sqrt{2[\ell(\hat{\alpha})-\ell(\alpha)]}=\operatorname{sgn}(\hat{\alpha}) \sqrt{2 \sum_{i=1}^{n} \log \left(\frac{p\left(x_{i} \mid \hat{\alpha}\right)}{p\left(x_{i} \mid \alpha\right)}\right)},
$$

so that it follows a standard normal distribution to third-order accuracy. This gives rise to the so-called Barndorff-Nielsen $R^{*}$ formula [4, sec. 6.6]:

$$
R_{\mathrm{LR}}^{*}(\alpha)=R_{\mathrm{LR}}(\alpha)+\frac{1}{R_{\mathrm{LR}}(\alpha)} \log \left|\frac{U(\alpha)}{R_{\mathrm{LR}}(\alpha)}\right|
$$

where $U(\alpha)$ is a quantity constructed from sample-space derivatives of $\ell(\alpha)$. The key idea behind this result is that for a random variable $X$ with a density of the form $h(x / \sqrt{n}) \phi(x)$, where $h \sim O(1)$, a normalizing transformation is: $X^{*}=X-X^{-1} \log h(X / \sqrt{n})$.

Explicit evaluation of $U(\alpha)$ is not possible for cases (such as ours) in which $\ell(\alpha)$ cannot be expressed in terms of the MLE, but an approximation based on covariances can be constructed [15, sec. 7.5]. However, this involves the computation of analytically intractable integrals. Another shortcoming of the $R^{*}$ formula is that it is only available for the LR statistic. Thus, if $R$ represents any of the statistics in Table 2, each of which possesses an Edgeworth expansion of the form (15) as shown under Strategy 1, we advocate usage of the normalized statistic (16). The resulting $\tilde{R}$ will follow a $\mathcal{N}(0,1)$ to an accuracy of $O\left(n^{-3 / 2}\right)$ under $\mathcal{H}_{0}$. However, it must be kept in mind that this is an asymptotic statement regarding the order of the error as $n$ grows. For a given $n$ the magnitude of the error will still be governed by an appropriate constant that could in some cases be quite large.

To quantify the deviations of $R$ from normality as a function of $r$, we investigate the quantity

$$
\Delta R(r)=r-\tilde{R}(r) .
$$

In combination with (15), the first order Taylor expansion of (14) yields a simple approximation $\Delta R(r) \approx \frac{a(r)}{\sqrt{n}}+\frac{b(r)}{n}$, valid to $O\left(n^{-1}\right)$. 
Without loss of generality, for the rest of the paper we will focus on the properties of the unnormalized $R$, but in an actual application it would behoove the practitioner to use the normalized $\tilde{R}$ version since computation of required quantiles for hypothesis testing will be straightforward. Important results such as type I and type II error rates studied in Section 6 for $R$ will be identical for $\tilde{R}$.

\section{An Illustrative Example}

In this section we choose a particular model in order to illustrate the consequences of applying the Edgeworth expansions for the statistics in Table 2. As choices for the background and signal under model (1), we select a relatively simple configuration by letting $b(x)$ follow a uniform distribution on $[0,1]$, and $s(x)$ a truncated Gaussian on $[0,1]$ :

$$
b(x)=\left\{\begin{array}{ll}
1, & \text { if } x \in[0,1] \\
0, & \text { if } x \notin[0,1]
\end{array}, \quad s(x)=\left\{\begin{array}{cl}
e^{-\frac{(x-\mu)^{2}}{2 \sigma^{2}}} / \int_{0}^{1} e^{-\frac{(y-\mu)^{2}}{2 \sigma^{2}}} d y, & \text { if } x \in[0,1] \\
0, & \text { if } x \notin[0,1]
\end{array} .\right.\right.
$$

This gives a flat background superimposed with a Gaussian signal. For this and the remaining sections, whenever specific settings of the signal are needed, we use

$$
\mu=0.5, \quad \text { and } \quad \sigma=0.1,
$$

implying that the values of the dimensionless parameters defined in (8) and (9) are $\gamma \approx 1.1$ and $\rho \approx 2.7$.

Figure 1 gives an idea of the impact of the signal location and scale parameters $\mu$ and $\sigma$ on $\gamma$ and $\rho$. In each respective panel, the value of the parameter not being varied is as specified in (20). While the curves for $\mu$ remain fairly flat, it is noteworthy that $\sigma$ has a dramatic effect on both quantities as it approaches the origin. This effect is better understood when we note that for small $\sigma$,

$$
\rho \approx \frac{\mathbb{E}_{s}\left[\frac{s^{3}}{b^{3}}\right]}{\left(\mathbb{E}_{s}\left[\frac{s}{b}\right]\right)^{2}}=\frac{1}{\sqrt{2 \pi}} \sigma^{-1}, \quad \text { and } \quad \gamma \approx \frac{\mathbb{E}_{s}\left[\frac{s^{2}}{b^{2}}\right]}{\left(\mathbb{E}_{s}\left[\frac{s}{b}\right]\right)^{3 / 2}}=\sqrt{\frac{2}{3}} \pi^{-1 / 4} \sigma^{-1 / 2}
$$

To give a sense of the effective normal approximation error for some of the statistics constructed from random samples of size $n$ from model (19)-(20), Figure 2 plots the value $\Delta R(r)$ defined in (18) as a function of the observed statistic value $r$, for $R_{\mathrm{W}}$ and $R_{\mathrm{LR}}$. (A standard normal distribution would have a deviation of $\Delta R(r)=0$.) The large deviations of the un-normalized $R_{\mathrm{W}}$ values from its third-order normal-corrected version are particularly striking, especially at the lower sample sizes. To understand what this means, note that the deviation of $R_{\mathrm{W}}$ at $r=5$ for $n=200$ is approximately $\Delta R_{\mathrm{W}}(5)=0.25$, which, for the $Z \sim \mathcal{N}(0,1)$ reference distribution under $\mathcal{H}_{0}$, translates into the $p$-value being smaller by a factor of $P(Z>5) / P(Z>5.25) \approx 3.8$ (a higher signal significance would be claimed than what is supported by the data).

In stark contrast to this is the (by comparison) exceptionally low normal approximation error of $R_{\mathrm{LR}}$, e.g., at $r=4$ for $n=200, \Delta R_{\mathrm{LR}}(4)=-0.005$, which translates into the $p$-value being off by only a factor of $P(Z>4) / P(Z>3.995) \approx 0.98$. A glance at Table 2 reveals a possible reason for the good performance of the LR statistic: the $O\left(n^{-3 / 2}\right)$ values for the 3rd and 4th cumulants are zero. Since these cumulants are precisely the ones appearing with the high order Hermite polynomials in (7), they can contribute substantially to the magnitude of the Edgeworth approximation at large values of $r$. It was conjectured in [14] that this effect may be largely responsible for the near-optimality of $R_{\mathrm{LR}}$. 

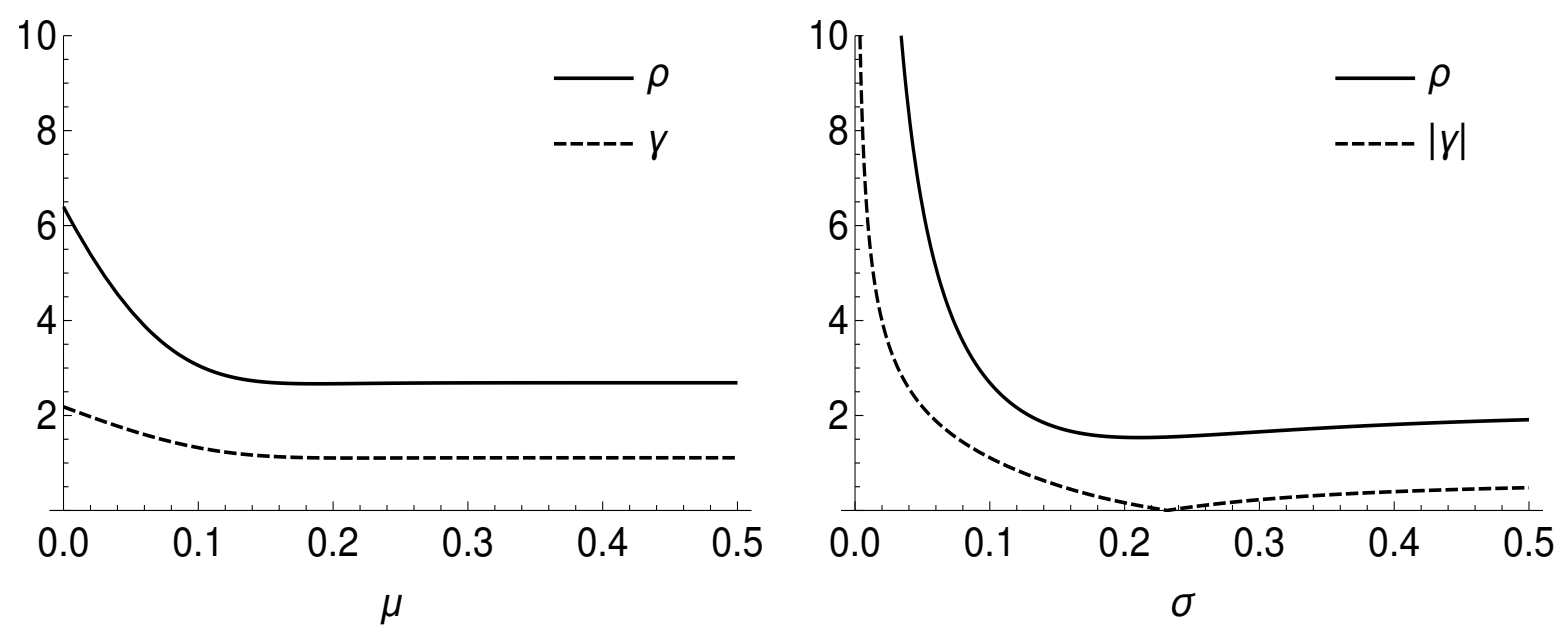

Figure 1: Impact of the signal location and scale parameters $\mu$ and $\sigma$ on the quantities $\gamma$ and $\rho$. In the left/right panel, the scale/location parameter is fixed at $\sigma=0.1 / \mu=0.5$.

\section{$5 \quad$ Numerical Simulations}

This section undertakes an extensive investigation of the properties of the statistics in Table 2. As the data generating process, we take the same flat background superimposed with Gaussian signal model as specified by (19) and (20). For each of the sample sizes $n=\{200,1000,5000,25000\}$, a total of $m=10^{9}$ pseudo-experiments (replications) are carried out whereby a dataset of size $n$ is independently drawn from the model (with $\alpha=0$ ) via Monte Carlo methods, in order to calculate the statistics in question.

Table 8 in Appendix D compares the (Edgeworth-predicted) analytical expected value of the statistic (first cumulant from Table 2) with its simulation-based empirical estimate (over the $m$ replications). The latter can be taken to be our best estimate of the true value; the extent to which it deviates from truth due to Monte Carlo error being quantified by the simulation uncertainty (in this case the standard error of the sample mean). Similar tables for the analytical standard deviation minus one, skewness, and excess kurtosis coefficients are given in Tables 9-11. If the statistics in question were exactly $\mathcal{N}(0,1)$, the simulated values would all be zero (to within simulation uncertainty). For the three classical statistics $R_{\mathrm{W}}, R_{\mathrm{LR}}$, and $R_{\mathrm{S}}$, the results are in excellent agreement with $O\left(n^{-3 / 2}\right)$ predictions for $n \geq 1000$; whereas for the remainder, values of $n \geq 25000$ are generally needed. Agreement with predictions is markedly worse for $R_{\mathrm{W} 4}$.

An alternative assessment via a chi-square goodness-of-fit test is carried out in Table 12 of Appendix D. The observed proportions of the respective $m=10^{9} R$ statistic values falling in each bin over the $m=10^{9}$ replications are compared with predicted probabilities for a $\mathcal{N}(0,1)$, and second and third order Edgeworth expansions. We see substantial disagreements with normality for all (unapproximated) statistics and all sample sizes. The agreement of simulations with Edgeworth predictions improves as one goes from $O\left(n^{-1}\right)$ to $O\left(n^{-3 / 2}\right)$, and from lower to higher $n$, particularly for $R_{\mathrm{W}}, R_{\mathrm{LR}}$, and $R_{\mathrm{S}}$.

Table 3 proceeds in similar fashion, but compares instead the Edgeworth-predicted tail probabilities for exceeding the value of $r=5$, a traditional threshold for signal discovery claims in high energy physics [12], with the corresponding simulation-based exceedances. Taking the case of table entries corresponding to $R_{\mathrm{W}}$ for example, the $O\left(n^{-3 / 2}\right)$ prediction is computed as $P\left(R_{\mathrm{W}}>5\right)$ 
Wald (Expected): $R_{W}$

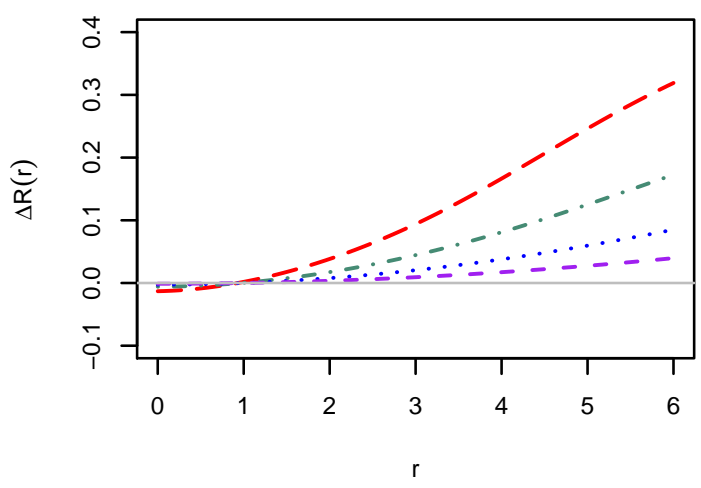

Score: $R_{S}$

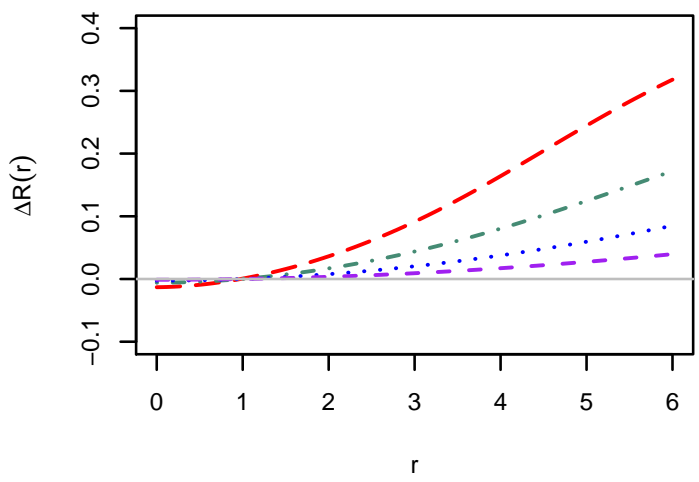

Wald (Observed): $R_{W 2}$

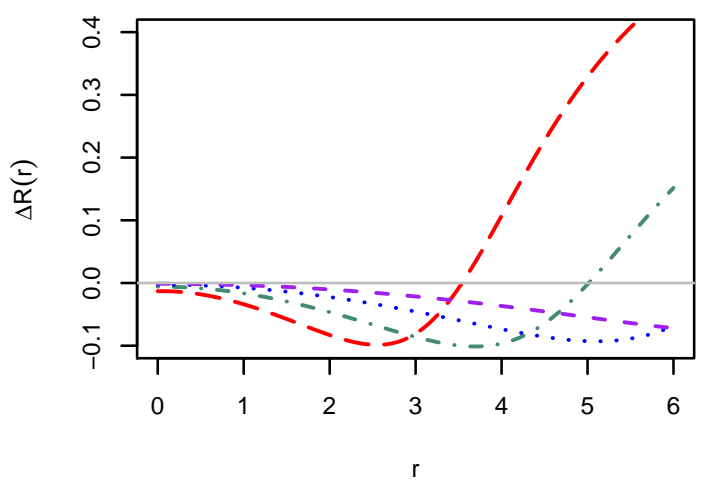

Likelihood Ratio: $\mathrm{R}_{\mathrm{LR}}$

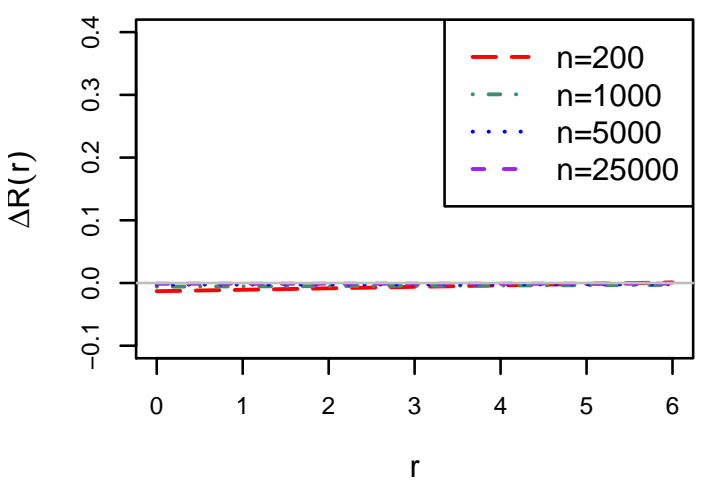

Figure 2: Effective normal approximation error $\Delta R(r)$ for the signed versions of the expected Wald $\left(R_{\mathrm{W}}\right)$, observed Wald $\left(R_{\mathrm{W}}\right)$, Score $\left(R_{\mathrm{S}}\right)$, and LR $\left(R_{\mathrm{LR}}\right)$ statistics, constructed from random samples of size $n$ from model (1) under signal and background densities (19) with $\mu=0.5$ and $\sigma=0.1$.

using (11), whereas the simulated value is the empirical proportion (say $\hat{p}$ ) of the $m=10^{9} R_{\mathrm{W}}$ values exceeding 5 . The simulation uncertainty is the standard error of the empirical proportion, $\sqrt{\hat{p}(1-\hat{p}) / m}$. Taking twice the simulation uncertainty as the metric (corresponding to $95 \%$ confidence), the last column indicates that predictions for $R_{\mathrm{W}}, R_{\mathrm{S}}$, and $R_{\mathrm{LR}}$ are generally in agreement with simulations (especially $R_{\mathrm{LR}}$ ), but the remaining statistics show significant differences at lower $n$. Consequently, it appears that one would need higher than third order based predictions in order to obtain nominally correct $p$-values for these cases. Finally, note that predictions for $R_{\mathrm{LR}}$ are also the closest to $\mathcal{N}(0,1)$ values, in agreement with the conclusions drawn from Figure 2.

Figure 3 presents the survival probabilities over the quantile range $4.5 \leq r \leq 5.5$ relevant for establishing credibility of signal discovery claims. The top panels show the effect of increasing the sample size from 200 to 1000, as well as increasing the order of the Edgeworth prediction from $O\left(n^{-1}\right)$ to $O\left(n^{-3 / 2}\right)$, for $R_{\mathrm{W}}$. Note that both predictions drift toward the $95 \%$ simulation uncertainty envelope (gray band) as $n$ increases; all of these converging to the $\mathcal{N}(0,1)$ line as expected. An interesting aberration occurs in the lower panels corresponding to $R_{\mathrm{W} 2}$ : not only is the $O\left(n^{-3 / 2}\right)$ prediction in the "wrong" direction, but the $O\left(n^{-1}\right)$ one became negative (and is thus 
Table 3: Comparison of Edgeworth-predicted survival probabilities at $r=5$ with simulation-based empirical exceedance proportions computed over $10^{9}$ replications. The corresponding $\mathcal{N}(0,1)$ value is $2.87 \times 10^{-7}$. Predictions that differ from simulated values by more than twice the simulation uncertainty appear in bold face.

\begin{tabular}{|c|c|c|c|c|}
\hline & $n$ & $\begin{array}{c}O\left(n^{-3 / 2}\right) \\
\text { Prediction }\end{array}$ & $\begin{array}{l}\text { Simulated } \\
\text { Value }\end{array}$ & $\begin{array}{l}\text { Simulation } \\
\text { Uncertainty }\end{array}$ \\
\hline \multirow{4}{*}{$R_{\mathrm{W}}$} & 200 & $9.98 \times 10^{-7}$ & $10.55 \times 10^{-7}$ & $0.32 \times 10^{-7}$ \\
\hline & 1000 & $5.44 \times 10^{-7}$ & $5.23 \times 10^{-7}$ & $0.23 \times 10^{-7}$ \\
\hline & 5000 & $3.90 \times 10^{-7}$ & $4.04 \times 10^{-7}$ & $0.20 \times 10^{-7}$ \\
\hline & 25000 & $3.30 \times 10^{-7}$ & $3.19 \times 10^{-7}$ & $0.18 \times 10^{-7}$ \\
\hline \multirow{4}{*}{$R_{\mathrm{W} 2}$} & 200 & $15.0 \times 10^{-7}$ & $0.61 \times 10^{-7}$ & $0.08 \times 10^{-7}$ \\
\hline & 1000 & $2.83 \times 10^{-7}$ & $0.90 \times 10^{-7}$ & $0.09 \times 10^{-7}$ \\
\hline & 5000 & $1.76 \times 10^{-7}$ & $1.63 \times 10^{-7}$ & $0.13 \times 10^{-7}$ \\
\hline & 25000 & $2.16 \times 10^{-7}$ & $2.10 \times 10^{-7}$ & $0.14 \times 10^{-7}$ \\
\hline \multirow{4}{*}{$R_{\mathrm{W} 3}$} & 200 & $15.1 \times 10^{-7}$ & $0.68 \times 10^{-7}$ & $0.08 \times 10^{-7}$ \\
\hline & 1000 & $2.86 \times 10^{-7}$ & $0.93 \times 10^{-7}$ & $0.10 \times 10^{-7}$ \\
\hline & 5000 & $1.77 \times 10^{-7}$ & $1.63 \times 10^{-7}$ & $0.13 \times 10^{-7}$ \\
\hline & 25000 & $2.16 \times 10^{-7}$ & $2.10 \times 10^{-7}$ & $0.14 \times 10^{-7}$ \\
\hline \multirow{4}{*}{$R_{\mathrm{W} 4}$} & 200 & $9.45 \times 10^{-7}$ & $0.71 \times 10^{-7}$ & $0.08 \times 10^{-7}$ \\
\hline & 1000 & $2.21 \times 10^{-7}$ & $1.07 \times 10^{-7}$ & $0.10 \times 10^{-7}$ \\
\hline & 5000 & $1.85 \times 10^{-7}$ & $1.87 \times 10^{-7}$ & $0.14 \times 10^{-7}$ \\
\hline & 25000 & $2.27 \times 10^{-7}$ & $2.26 \times 10^{-7}$ & $0.15 \times 10^{-7}$ \\
\hline \multirow{4}{*}{$R_{\mathrm{LR}}$} & 200 & $2.85 \times 10^{-7}$ & $2.82 \times 10^{-7}$ & $0.17 \times 10^{-7}$ \\
\hline & 1000 & $2.81 \times 10^{-7}$ & $2.70 \times 10^{-7}$ & $0.16 \times 10^{-7}$ \\
\hline & 5000 & $2.83 \times 10^{-7}$ & $2.94 \times 10^{-7}$ & $0.17 \times 10^{-7}$ \\
\hline & 25000 & $2.85 \times 10^{-7}$ & $2.79 \times 10^{-7}$ & $0.17 \times 10^{-7}$ \\
\hline \multirow{4}{*}{$R_{\mathrm{S}}$} & 200 & $9.90 \times 10^{-7}$ & $10.26 \times 10^{-7}$ & $0.32 \times 10^{-7}$ \\
\hline & 1000 & $5.43 \times 10^{-7}$ & $4.82 \times 10^{-7}$ & $0.22 \times 10^{-7}$ \\
\hline & 5000 & $3.89 \times 10^{-7}$ & $3.59 \times 10^{-7}$ & $0.19 \times 10^{-7}$ \\
\hline & 25000 & $3.30 \times 10^{-7}$ & $3.18 \times 10^{-7}$ & $0.18 \times 10^{-7}$ \\
\hline
\end{tabular}

absent from the plots). Such unexpected behavior far out in the tails, as well as the possibility for a lower order approximation to be more accurate than a higher order one, are documented in the literature $[15$, sec. 5.3].

Figure 4 complements Figure 3 by displaying the corresponding results for $R_{\mathrm{LR}}$ and $R_{\mathrm{S}}$. As noted earlier, the results for the former statistic are remarkable in terms of: (i) close agreement with the $\mathcal{N}(0,1)$ line, and (ii) the relatively small magnitude of the Edgeworth corrections. The behavior of $R_{\mathrm{S}}$ on the other hand is similar to that of $R_{\mathrm{W}}$.

\section{Study of Type I and Type II Errors}

This section undertakes a study of the type I and type II error rates for testing the null $\mathcal{H}_{0}$ vs. the alternative $\mathcal{H}_{1}$. In the context of signal detection, these errors would correspond to false positive and false negative probabilities, respectively. Throughout the study we fix the nominal (or target) probability of type I error at $q_{0} \equiv 1-\Phi(5) \approx 2.87 \times 10^{-7}$. With the model settings once again 

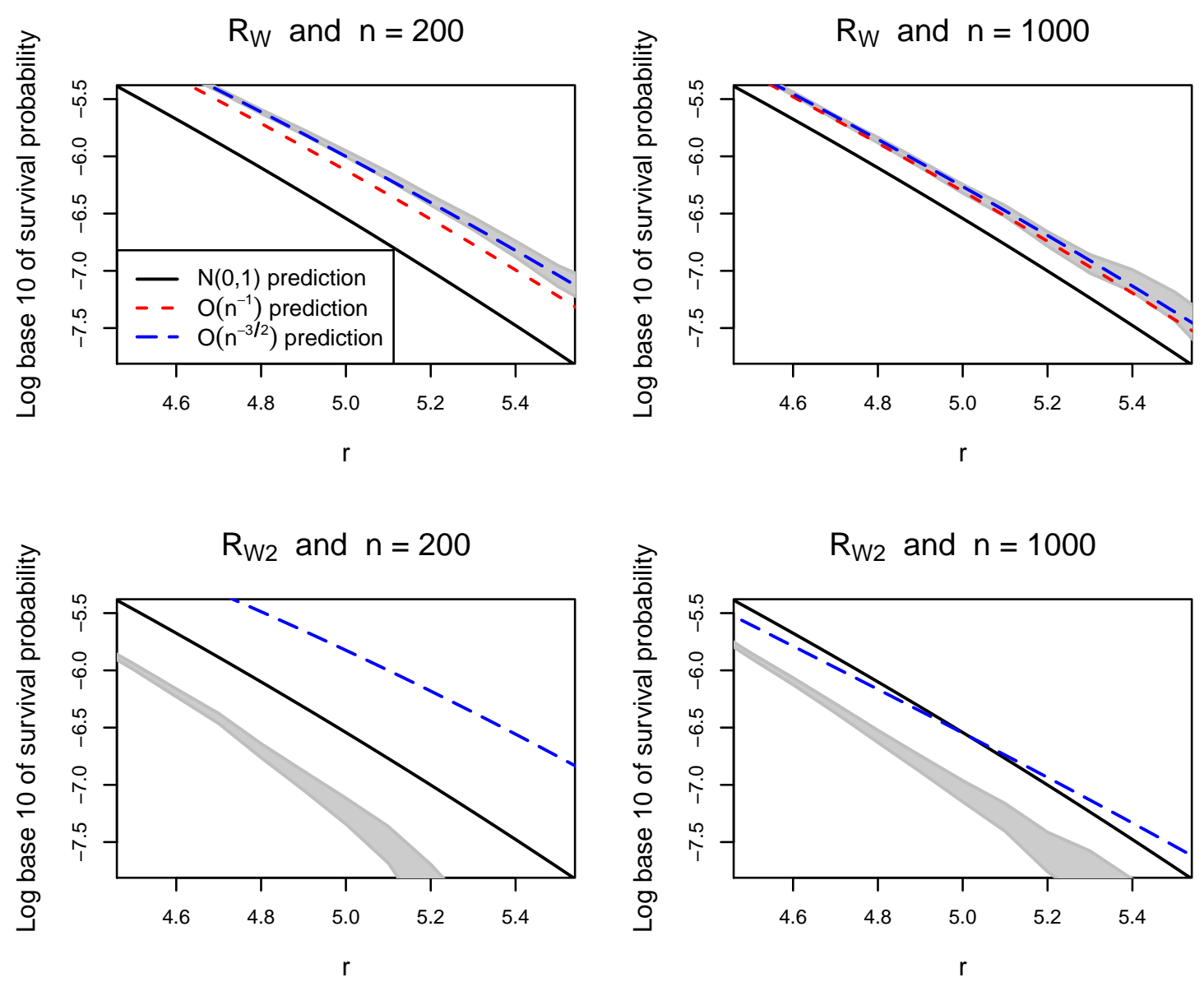

Figure 3: Second and third order approximated Edgeworth log survival probabilities of $R_{\mathrm{W}}$ (top panels) and $R_{\mathrm{W} 2}$ (bottom panels), computed at quantiles $r$ for $n=200$ (left panels) and $n=1000$ (right panels). The gray band is a $95 \%$ simulation uncertainty envelope, corresponding to the true survival probability.

specified by (19) and (20), we generate $m=10^{9}$ replicates, each comprised of a random sample of size $n$ from the model. To characterize the error rates, we introduce the following notation: $c_{\text {pre }}(n)$ is the quantile which corresponds to the predicted survival function value $q_{0}$. For any statistic $R$ in Table 2, this quantile is the solution of the equation $q_{0}=P_{\text {pre }}\left(R>c_{\text {pre }}(n)\right)$, where $P_{\text {pre }}(\cdot)$ is the probability predicted by the Edgeworth expansions of section 3.1. Then, assuming that $\mathcal{H}_{0}$ is rejected if $R>c_{\text {pre }}(n)$,

$$
q_{1}(n) \equiv P_{\text {sim }}\left(R>c_{\text {pre }}(n) \mid \alpha=0\right)
$$

is the probability of a type I error (i.e., of falsely rejecting $\mathcal{H}_{0}$ given that the true model has $\alpha=0$ ), estimated from simulations. Additionally, denote by $c_{\operatorname{sim}}(n)$ the quantile value established using the empirical survival function determined via simulations. That is, $c_{\operatorname{sim}}(n)$ is the solution of the equation $q_{0}=P_{\text {sim }}\left(R>c_{\text {sim }}(n) \mid \alpha=0\right)$.

Table 4 gives the values of the predicted and simulated, $c_{\text {pre }}(n)$ and $c_{\text {sim }}(n),\left(1-q_{0}\right)$-th quantiles. The uncertainty here is approximated by the asymptotic standard error of the empirical $\left(1-q_{0}\right)$ - 

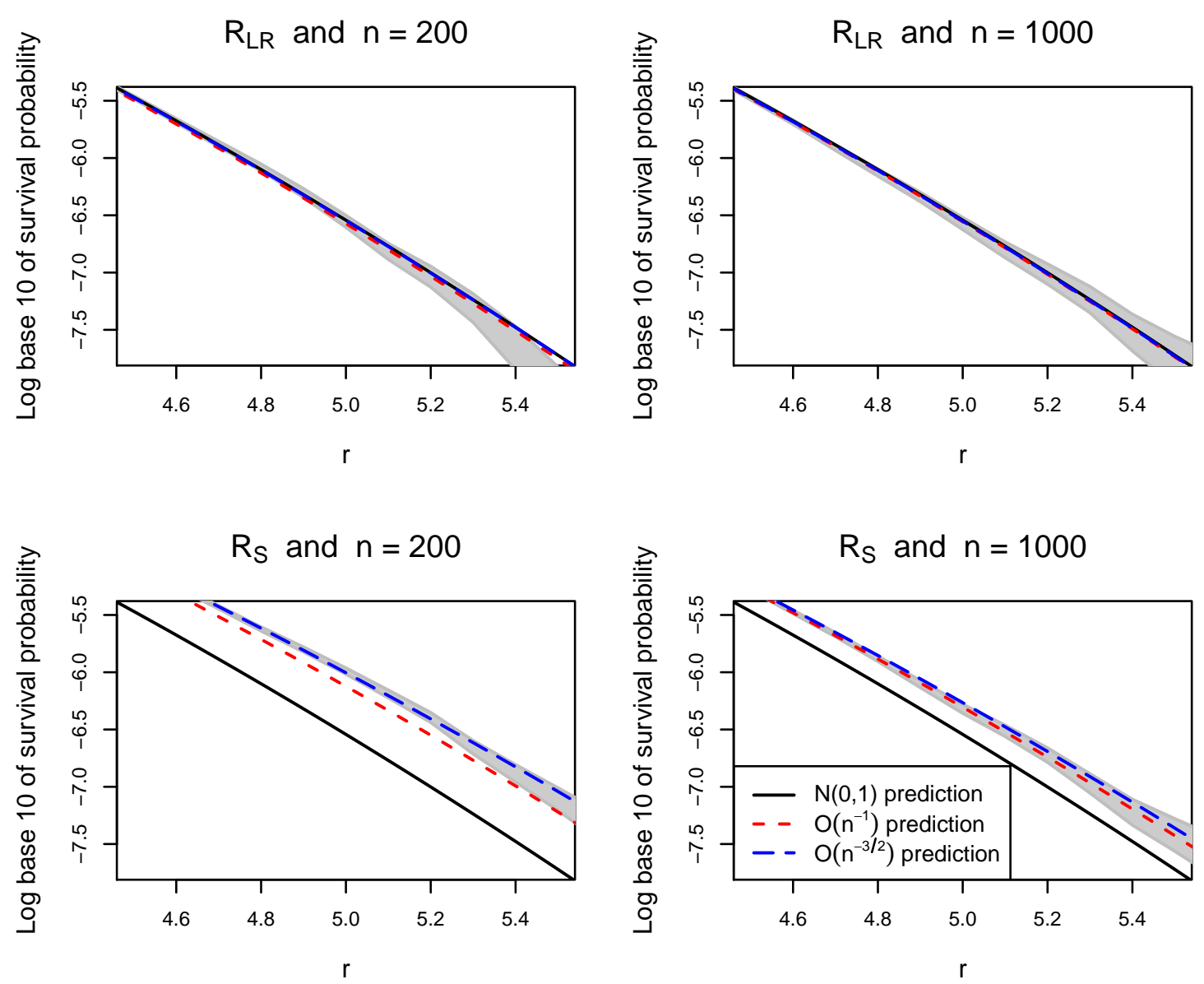

Figure 4: Second and third order approximated Edgeworth log survival probabilities of $R_{\mathrm{LR}}$ (top panels) and $R_{\mathrm{S}}$ (bottom panels), computed at quantiles $r$ for $n=200$ (left panels) and $n=1000$ (right panels). The gray band is a $95 \%$ simulation uncertainty envelope, corresponding to the true survival probability.

th quantile, given by $\sqrt{\left(1-q_{0}\right) q_{0} /\left[m f\left(c_{\text {pre }}(n)\right)^{2}\right]}$, where $f(z)=-d S_{\text {pre }}(z) / d z$. Since this density function is only available for the predicted quantiles, we attach the uncertainty to these values in the form of plus and minus the standard error.

The $c_{\text {pre }}(n)$ predictions from Table 4 can now be used as the basis for a type I and II error assessment. We start by investigating the nominal type I error probability $q_{1}(n)$ as defined in $(21)$. These results, computed empirically based on the $m$ Monte Carlo replicates, are presented in Table 5. If the $O\left(n^{-3 / 2}\right)$ predictions were exact, we would expect approximately $95 \%$ of all values to be within twice the simulation uncertainty of $0.17 \times 10^{-7}$ from the nominal value of $q_{0}$. We once again note that the three classical statistics, $R_{\mathrm{W}}, R_{\mathrm{LR}}$, and $R_{\mathrm{S}}$, are the only ones to perform at nominal levels (to within Monte Carlo uncertainty) for all sample sizes.

For the statistical power study, we fix the probability of type I error at $q_{0}$, and determine the probability of type II error, denoted by $q_{2}\left(n, \alpha_{1}\right)=P\left(R \leq c_{\text {pre }}(n) \mid \alpha=\alpha_{1}\right)$. Implicit in the notation is the fact that $q_{2}(\cdot)$ will vary with both $n$ and the actual model value for the signal fraction of $\alpha=\alpha_{1}$ under $\mathcal{H}_{1}$. (Also note that $q_{1}(n)=1-q_{2}(n, 0)$.) For each $n$, we set the signal fraction at 
Table 4: $O\left(n^{-3 / 2}\right)$ Edgeworth predictions for the $\left(1-q_{0}\right)$-th quantiles of the statistics in Table 2, compared to their corresponding values computed based on $10^{9}$ simulations. The uncertainty in the predicted values appends plus and minus the standard error for the empirical quantile. Predictions that deviate by more than twice the uncertainty from their simulated values are bolded.

\begin{tabular}{|c|l|cccc|}
\hline \multirow{2}{*}{ Statistic } & \multirow{4}{*}{ Method } & $n=200$ & $n=1000$ & $n=5000$ & $n=25000$ \\
\hline \multirow{2}{*}{$R_{\mathrm{W}}$} & Predicted & $5.267 \pm 0.012$ & $5.131 \pm 0.012$ & $5.061 \pm 0.012$ & $5.028 \pm 0.012$ \\
& Simulated & 5.286 & 5.123 & 5.046 & 5.028 \\
\hline \multirow{2}{*}{$R_{\mathrm{W} 2}$} & Predicted & $\mathbf{5 . 3 9 2} \pm \mathbf{0 . 0 1 3}$ & $\mathbf{4 . 9 9 8} \pm \mathbf{0 . 0 1 3}$ & $\mathbf{4 . 9 0 8} \pm \mathbf{0 . 0 1 1}$ & $4.946 \pm 0.011$ \\
& Simulated & 4.736 & 4.790 & 4.872 & 4.943 \\
\hline \multirow{2}{*}{$R_{\mathrm{W} 3}$} & Predicted & $\mathbf{5 . 3 9 4} \pm \mathbf{0 . 0 1 3}$ & $\mathbf{5 . 0 0 0} \pm \mathbf{0 . 0 1 3}$ & $\mathbf{4 . 9 0 8} \pm \mathbf{0 . 0 1 1}$ & $4.946 \pm 0.011$ \\
& Simulated & 4.744 & 4.791 & 4.872 & 4.944 \\
\hline \multirow{2}{*}{$R_{\mathrm{W} 4}$} & Predicted & $\mathbf{5 . 2 9 2} \pm \mathbf{0 . 0 1 4}$ & $\mathbf{4 . 9 4 5} \pm \mathbf{0 . 0 1 3}$ & $\mathbf{4 . 9 1 8} \pm \mathbf{0 . 0 1 1}$ & $4.956 \pm 0.011$ \\
& Simulated & 4.755 & 4.822 & 4.893 & 4.954 \\
\hline \multirow{2}{*}{$R_{\mathrm{LR}}$} & Predicted & $4.999 \pm 0.011$ & $4.996 \pm 0.011$ & $4.998 \pm 0.011$ & $4.999 \pm 0.011$ \\
& Simulated & 4.990 & 4.988 & 4.983 & 4.998 \\
\hline \multirow{2}{*}{$R_{\mathrm{S}}$} & Predicted & $5.265 \pm 0.012$ & $5.131 \pm 0.012$ & $5.061 \pm 0.012$ & $5.028 \pm 0.012$ \\
& Simulated & 5.259 & 5.121 & 5.050 & 5.026 \\
\hline
\end{tabular}

Table 5: Type I error probabilities $q_{1}(n)$ as defined in (21). The survival function values are obtained based on $10^{9}$ simulated replicates for the predicted quantiles $c_{\text {pre }}(n)$ from Table 4 . Values that deviate by more than twice the simulation uncertainty of $0.17 \times 10^{-7}$ from the nominal value of $q_{0}=2.87 \times 10^{-7}$ are bolded.

\begin{tabular}{|r|cccccc|}
\hline \multicolumn{1}{|c}{$n$} & $R_{\mathrm{W}}$ & $R_{\mathrm{W} 2}$ & $R_{\mathrm{W} 3}$ & $R_{\mathrm{W} 4}$ & $R_{\mathrm{LR}}$ & $R_{\mathrm{S}}$ \\
\hline 200 & $3.03 \times 10^{-7}$ & $\mathbf{0 . 0 7} \times \mathbf{1 0}^{-\mathbf{7}}$ & $\mathbf{0 . 0 7} \times \mathbf{1 0}^{-\mathbf{7}}$ & $\mathbf{0 . 1 3} \times \mathbf{1 0}^{-\mathbf{7}}$ & $2.68 \times 10^{-7}$ & $2.73 \times 10^{-7}$ \\
1000 & $2.71 \times 10^{-7}$ & $\mathbf{0 . 7 8} \times \mathbf{1 0}^{-\mathbf{7}}$ & $\mathbf{0 . 7 8} \times \mathbf{1 0}^{-\mathbf{7}}$ & $\mathbf{1 . 3 5} \times \mathbf{1 0}^{-\mathbf{7}}$ & $2.77 \times 10^{-7}$ & $2.72 \times 10^{-7}$ \\
5000 & $2.65 \times 10^{-7}$ & $\mathbf{2 . 4 4} \times \mathbf{1 0}^{-\mathbf{7}}$ & $\mathbf{2 . 4 4} \times \mathbf{1 0}^{-\mathbf{7}}$ & $2.56 \times 10^{-7}$ & $2.72 \times 10^{-7}$ & $2.77 \times 10^{-7}$ \\
25000 & $2.87 \times 10^{-7}$ & $2.82 \times 10^{-7}$ & $2.82 \times 10^{-7}$ & $2.83 \times 10^{-7}$ & $2.83 \times 10^{-7}$ & $2.86 \times 10^{-7}$ \\
\hline
\end{tabular}

$\alpha_{1}=5 \sigma_{\hat{\alpha}}$, where $\sigma_{\hat{\alpha}}=I(0)^{-1 / 2}$ is the Cramer-Rao uncertainty of $\alpha$ under $\mathcal{H}_{0}$ (i.e., the asymptotic standard error of the MLE for true $\alpha=0)$. The values of $\sigma_{\hat{\alpha}}$ for the $s(x)$ and $b(x)$ used in this study are given in Table 6 .

Table 6: Values of the Cramer-Rao uncertainty for $\mathcal{H}_{0}, \sigma_{\hat{\alpha}}$, and corresponding values of $\alpha=\alpha_{1}$ used as the actual model signal fraction under $\mathcal{H}_{1}$.

\begin{tabular}{|c|c|c|}
\hline \multicolumn{1}{|c|}{$n$} & $\sigma_{\hat{\alpha}}$ & $\alpha_{1}=5 \sigma_{\hat{\alpha}}$ \\
\hline 200 & $5.2401 \times 10^{-2}$ & 0.26200 \\
1000 & $2.3434 \times 10^{-2}$ & 0.11717 \\
5000 & $1.0480 \times 10^{-2}$ & 0.05240 \\
25000 & $0.4687 \times 10^{-2}$ & 0.02343 \\
\hline
\end{tabular}


Table 7 gives the resulting probability of type II error. These are determined empirically from the simulations by computing the proportion of samples with $\hat{\alpha}$ below the corresponding predicted quantiles $c_{\text {pre }}(n)$, as well as the simulated quantiles $c_{\operatorname{sim}}(n)$, listed in Table 4 . As before, there is close agreement between "predicted" and "simulated" values at all sample sizes for the three classical statistics, $R_{\mathrm{W}}, R_{\mathrm{LR}}$, and $R_{\mathrm{S}}$. Note that the nominal $q_{2}\left(n, \alpha_{1}\right)$ value here is $q_{2}\left(\infty, \alpha_{1}\right)=0.5$, this being the value that all statistics converge to as $n \rightarrow \infty$. In line with earlier results, it is not surprising that $R_{\mathrm{LR}}$ yields the smallest predicted type II errors at each setting of $n$.

Table 7: Type II error probabilities for testing $\mathcal{H}_{1}$ with the quantiles listed in Table 4 , determined empirically from $10^{9}$ simulations with true $\alpha=\alpha_{1}$ as given in Table 6 . The smallest predicted value at each $n$ appears in bold. The simulation uncertainty in these results is at most $2 \times 10^{-5}$.

\begin{tabular}{|c|l|cccc|}
\hline \multirow{2}{*}{ Statistic } & \multicolumn{4}{|c}{ Sample Size $(n)$} \\
& Quantile & 200 & 1000 & 5000 & 25000 \\
\hline \multirow{2}{*}{$R_{\mathrm{W}}$} & Predicted & 0.59592 & 0.55021 & 0.52433 & 0.51130 \\
& Simulated & 0.60241 & 0.54701 & 0.51848 & 0.51143 \\
\hline \multirow{2}{*}{$R_{\mathrm{W} 2}$} & Predicted & 0.80891 & 0.63069 & 0.53250 & 0.51192 \\
& Simulated & 0.58540 & 0.54601 & 0.51775 & 0.51074 \\
\hline \multirow{2}{*}{$R_{\mathrm{W} 3}$} & Predicted & 0.80695 & 0.63082 & 0.53257 & 0.51193 \\
& Simulated & 0.58535 & 0.54601 & 0.51775 & 0.51074 \\
\hline \multirow{2}{*}{$R_{\mathrm{W} 4}$} & Predicted & 0.77782 & 0.59609 & 0.52830 & 0.51156 \\
& Simulated & 0.58515 & 0.54577 & 0.51778 & 0.51074 \\
\hline \multirow{2}{*}{$R_{\mathrm{LR}}$} & Predicted & $\mathbf{0 . 5 8 8 7 3}$ & $\mathbf{0 . 5 4 8 2 7}$ & $\mathbf{0 . 5 2 3 8 5}$ & $\mathbf{0 . 5 1 1 1 9}$ \\
& Simulated & 0.58533 & 0.54501 & 0.51796 & 0.51074 \\
\hline \multirow{2}{*}{$R_{\mathrm{S}}$} & Predicted & 0.59510 & 0.55011 & 0.52432 & 0.51129 \\
& Simulated & 0.59296 & 0.54672 & 0.52024 & 0.51059 \\
\hline
\end{tabular}




\section{Discussion}

A wide gamut of near-optimal statistics can be used in testing for the presence of a signal under a mixture model with unknown signal fraction. The focus of the study presented in this manuscript was on computing Edgeworth approximations to $p$-values of the asymptotic distributions of such statistics under the null hypothesis of no signal, so as to provide more accurate inferences for finite samples. Comparisons were made between approximations of different orders, highlighting striking deviations from the target standard normal reference distribution in some cases, with consequent $p$-value inflation/deflation. Finally, the performance of the corrected statistics was examined in terms of false positive and false negative signal detection error rates.

Given the insights gained from this study, the following summary remarks can be offered. For small and moderate sample sizes, deviations from normality for some likelihood-based statistics used in signal searches, in particular the observed version of Wald and its variants, can be significant, thus altering the false discovery error rate. Moreover, for narrow signals these deviations are unbounded. The third-order approximated versions of these statistics manifest a substantially improved agreement with the behavior found in simulations.

It is desirable to assess the magnitude of these deviations from normality. Their influence can be quantified by comparing the approximations developed in this study, to various orders of accuracy, with the asymptotic formulae, for all possible nuisance parameter values of the model. If the finite sample effects are substantial, proper signal strength $p$-values should then be established by direct simulations.

In comparison with the Wald and Score tests, deviations from normality are substantially milder for the signed LR statistic. Tests based on the likelihood ratio should therefore be preferred. The latter also have better capabilities of detecting actual signals (higher power), while still maintaining the false discovery rate at acceptably low levels. As speculated by [14] who proves that the $k$-th cumulant of $R_{\mathrm{LR}}$ vanishes to $O\left(n^{-k / 2}\right)$ for all $k \geq 3$, this fact "... would seem to be the main asymptotic property governing the accuracy behavior..." of $R_{\mathrm{LR}}$. To further elucidate this statement, we derive the fourth order Edgeworth-approximated tail probabilities for $R_{\mathrm{LR}}$ in Appendix C. As predicted, the $\kappa_{3}$ value is $O\left(n^{-3 / 2}\right)$ while $\kappa_{4}=\kappa_{5}=0$ to $O\left(n^{-2}\right)$. The vanishing of the highest order cumulants results in the suppression of high order Hermite polynomials in the Edgeworth series, $H_{k}(z)$ with $k>2$, and it is precisely these terms that affect the tail behavior the most. Other statistics do not enjoy this property, containing terms up to $H_{8}(z)$ in their $O\left(n^{-2}\right)$ Edgeworth expansions.

In the cases when there are no nuisance parameters in $b(x)$, or the practitioner is willing to treat all nuisance parameters via the methodology of random fields (i.e., the Gross-Vitells method), $O\left(n^{-3 / 2}\right)$ normalized versions of the likelihood-based statistics via transformation (14) are suggested, facilitating standard inferences. Alternatively, the global significance of the signal test statistic could be adjusted conservatively, leading to a subsequent (conservative) estimate of the global $p$-value, by proceeding as follows:

- Let $\Delta R(r(\theta))$ denote the normal approximation error defined for each observed (local) $r \equiv$ $r(\theta)$ as in (18). Note that there is now an explicit dependence of $r$ (and hence $\Delta R$ ) on the nuisance parameter vector $\theta$.

- Perform a search over $\theta$ in order to locate the value of $\theta^{*}=\arg \max \Delta R(r(\theta))$. This search can utilize the same grid as the necessary search for $\hat{\theta}=\arg \max r(\theta)$. Note that $r(\hat{\theta})$ corresponds to the signal with the highest local significance.

- Calculate the global significance of the signal. This is evaluated in the $\mathcal{N}(0,1)$ approximation 
via the Gross-Vitells method, and is expressed in terms of the global $r[7,17]$.

- Adjust the above global $r$ by subtracting $\Delta R\left(r\left(\theta^{*}\right)\right)$. The global $p$-value is then estimated from the adjusted $r$ according to a $\mathcal{N}(0,1)$.

\section{Acknowledgments}

I. Volobouev thanks the Statistics Committee of the CMS Collaboration for comments and productive discussions. This work was supported in part by the United States Department of Energy grant DE-SC001592.

\section{References}

[1] Adler, R.J. (1981), The Geometry of Random Fields, New York: Wiley.

[2] Adler, R.J. Taylor, J.E. (2007), Random Fields and Geometry, New York: Springer.

[3] Cowan, G., Cranmer, K. Gross, E. and Vitells, O. (2011), "Asymptotic formulae for likelihoodbased tests of new physics", The European Physical Journal C, 71, 1554.

[4] Cox, D.R. and Barndorff-Nielsen, O.E. (1994), Inference and Asymptotics, London: Chapman \& Hall/CRC.

[5] Davies, R.B. (1987), "Hypothesis testing when a nuisance parameter is present only under the alternative", Biometrika, 74 33-43.

[6] Eadie, W.T., Drijard, D., James, F.E., Roos, M. and Sadoulet, B. (1971), Statistical Methods in Experimental Physics, Amsterdam: North-Holland.

[7] Gross, E. and Vitells, O. (2010), "Trial factors for the look elsewhere effect in high energy physics", The European Physical Journal C, 70, 525-530.

[8] James, F. and Roos, M. (1975), "Minuit - a system for function minimization and analysis of the parameter errors and correlations", Computer Physics Communications 10, 343-367.

[9] Joanes, D.N. and Gill, C.A. (1998), "Comparing measures of sample skewness and kurtosis", Journal of the Royal Statistical Society, Series D (The Statistician), 47, 183-189.

[10] Lehmann, E. and Romano, J. (2005), Testing Statistical Hypotheses, 3rd ed., New York: Springer.

[11] Lyons, L. (2008), "Open Statistical Issues in Particle Physics", The Annals of Applied Statistics, 2, 887-915.

[12] Lyons, L. (2013), "Discovering the Significance of 5 sigma", arXiv:1310.1284 [physics.data-an].

[13] McLachlan, G. and Peel, D. (2000), Finite Mixture Models, Hoboken: Wiley.

[14] Mykland, P.A. (1999), "Bartlett identities and large deviations in likelihood theory", The Annals of Statistics, 27, 1105-1117.

[15] Severini, T. (2000), Likelihood Methods in Statistics, New York: Oxford University Press. 
[16] Shao, J. (2003), Mathematical Statistics, 2nd ed., New York: Springer.

[17] Vitells, O. and Gross, E. (2011), "Estimating the significance of a signal in a multi-dimensional search", Astroparticle Physics, 35, 230-234.

[18] Wilks, S (1938), "The large-sample distribution of the likelihood ratio for testing composite hypotheses", The Annals of Mathematical Statistics, 9, 60-62.

\section{A Calculation of Cumulants of the Log-Likelihood Function}

Recalling that $\mathbb{E}[\cdot]$ and $\mathbb{V}[\cdot]$ denote, respectively, expectation and variance under $b(x)$, and that $n \nu_{i j k l}$ represents the $(i, j, k, l)$ joint cumulant of $\left(\ell_{1}(0), \ldots, \ell_{4}(0)\right)$, we compute initially

$$
n \nu_{1}=\mathbb{E}\left[\ell_{1}(0)\right]=\mathbb{E}\left[\sum_{i=1}^{n} \frac{s\left(x_{i}\right)-b\left(x_{i}\right)}{b\left(x_{i}\right)}\right]=\sum_{i=1}^{n} \int s(x) d x-\sum_{i=1}^{n} \int b(x) d x=n-n=0,
$$

whence $\nu_{1}=0$. Similar calculations now yield:

- $\nu_{2}=\frac{1}{n} \mathbb{E}\left[\ell_{1}(0)^{2}\right]=\mathbb{E}_{s}[s / b]-1$.

- $\nu_{3}=\frac{1}{n} \mathbb{E}\left[\ell_{1}(0)^{3}\right]=\mathbb{E}_{s}\left[s^{2} / b^{2}\right]-3 \mathbb{E}_{s}[s / b]+2=\gamma \nu_{2}^{3 / 2}$.

- $\nu_{11}=\frac{1}{n} \mathbb{E}\left[\ell_{1}(0) \ell_{2}(0)\right]=-\gamma \nu_{2}^{3 / 2}$.

- $\nu_{001}=\frac{1}{n} \mathbb{E}\left[\ell_{3}(0)\right]=2 \gamma \nu_{2}^{3 / 2}$.

- $\nu_{101}=\frac{1}{n} \mathbb{E}\left[\ell_{1}(0) \ell_{3}(0)\right]=2\left[\mathbb{E}_{s}\left(s^{3} / b^{3}\right)-4 \mathbb{E}_{s}\left(s^{2} / b^{2}\right)+6 \mathbb{E}_{s}(s / b)-3\right]=2 \rho \nu_{2}^{2}$.

- $\nu_{4}=\frac{1}{n} \mathbb{E}\left[\ell_{1}(0)^{4}\right]-\frac{3}{n}\left[\mathbb{E} \ell_{1}(0)^{2}\right]^{2}=(\rho-3) \nu_{2}^{2}$.

- $\nu_{0001}=\frac{1}{n} \mathbb{E}\left[\ell_{4}(0)\right]=-6 \rho \nu_{2}^{2}$.

- $\nu_{02}=\frac{1}{n} \mathbb{V}\left[\ell_{2}(0)\right]=(\rho-1) \nu_{2}^{2}$.

- $\nu_{21}=\frac{1}{n} \mathbb{E}\left[\ell_{1}(0)^{2} \ell_{2}(0)\right]-\frac{1}{n} \mathbb{E}\left[\ell_{1}(0)^{2}\right] \mathbb{E}\left[\ell_{2}(0)\right]=(1-\rho) \nu_{2}^{2}$.

These results use the fact that the expressions for $\gamma$ and $\rho$ defined in (8) and (9) become, $\gamma=\nu_{3} / \nu_{2}^{3 / 2}$, and $\rho-3=\nu_{4} / \nu_{2}^{2}$. Straightforward computations also yield the following expressions for the information numbers under model (1):

$$
J(\alpha)=\sum_{i=1}^{n} \frac{\left(s\left(x_{i}\right)-b\left(x_{i}\right)\right)^{2}}{p\left(x_{i} \mid \alpha\right)^{2}}, \quad \text { and } \quad I(\alpha)=n \mathbb{E}_{s}\left[\frac{(s(x)-b(x))^{2}}{s(x) p(x \mid \alpha)}\right] .
$$

\section{B Third-Order Edgeworth Expansions for $R_{\mathrm{W} 3}$ and $R_{\mathrm{W} 4}$}

For $R_{\mathrm{W} 3}$, substitution of the cumulants in Table 2 into (7) gives:

$$
\begin{aligned}
P\left(R_{\mathrm{W} 3} \leq z\right)=\Phi(z) & -\phi(z)\left[n^{-1 / 2} \gamma\left(-\frac{1}{2}-\frac{1}{3} H_{2}(z)\right)\right. \\
& \left.+n^{-1}\left(\frac{1}{36}\left(63 \rho-28 \gamma^{2}\right) z+\frac{1}{12}\left(5 \rho+2 \gamma^{2}\right) H_{3}(z)+\frac{\gamma^{2}}{18} H_{5}(z)\right)+O\left(n^{-3 / 2}\right)\right] .
\end{aligned}
$$


For $R_{\mathrm{W} 4}$, the second cumulant behavior differs from the other cases considered in that $\kappa_{2}=$ $1+O\left(n^{-1 / 2}\right)$, so that the Edgeworth expansion in (7) must be rederived:

$$
\begin{aligned}
F(z)=\Phi(z)-\phi(z)\left[\kappa_{1}+\right. & \left(\frac{1}{6} \kappa_{3}+\frac{1}{2} \kappa_{1}\left(\kappa_{2}-1\right)\right) H_{2}(z)+\frac{1}{2}\left(\kappa_{1}^{2}+\kappa_{2}-1\right) z \\
& +\left(\frac{1}{6} \kappa_{1} \kappa_{3}+\frac{1}{24} \kappa_{4}+\frac{1}{8}\left(\kappa_{2}-1\right)^{2}\right) H_{3}(z) \\
& \left.+\frac{1}{12}\left(\kappa_{2}-1\right) \kappa_{3} H_{4}(z)+\frac{1}{72} \kappa_{3}^{2} H_{5}(z)+O\left(n^{-3 / 2}\right)\right] .
\end{aligned}
$$

Substitution of the cumulants in Table 2 into this expression then yields:

$$
\begin{aligned}
& P\left(R_{\mathrm{W} 4} \leq z\right)=\Phi(z)-\phi(z)\left[n^{-1 / 2} \gamma\left(\frac{z}{3}-\frac{1}{2}-\frac{1}{3} H_{2}(z)\right)\right. \\
&+n^{-1}\left(\frac{\gamma^{2}}{3}-\rho+\frac{1}{36}\left(63 \rho-22 \gamma^{2}\right) z-\frac{2}{3} \rho H_{2}(z)\right. \\
&\left.\left.\quad+\frac{1}{36}\left(15 \rho+8 \gamma^{2}\right) H_{3}(z)-\frac{\gamma^{2}}{9} H_{4}(z)+\frac{\gamma^{2}}{18} H_{5}(z)\right)+O\left(n^{-3 / 2}\right)\right] .
\end{aligned}
$$

\section{Fourth-Order Edgeworth Expansion for $R_{\mathrm{LR}}$}

We extend (7) by computing the $O\left(n^{-2}\right)$ Edgeworth expansion for the normal density:

$$
\begin{aligned}
F(z)= & \Phi(z)-\phi(z)\left[\kappa_{1}+\frac{1}{2}\left(\kappa_{1}^{2}+\kappa_{2}-1\right) z+\left(\frac{1}{6}\left(\kappa_{1}^{3}+\kappa_{3}\right)+\frac{1}{2} \kappa_{1}\left(\kappa_{2}-1\right)\right) H_{2}(z)\right. \\
& +\left(\frac{1}{6} \kappa_{1} \kappa_{3}+\frac{1}{24} \kappa_{4}\right) H_{3}(z)+\left(\frac{1}{12} \kappa_{3}\left(\kappa_{1}^{2}+\kappa_{2}-1\right)+\frac{1}{24} \kappa_{1} \kappa_{4}+\frac{1}{120} \kappa_{5}\right) H_{4}(z) \\
& \left.+\frac{1}{72} \kappa_{3}^{2} H_{5}(z)+\frac{1}{144} \kappa_{3}\left(2 \kappa_{1} \kappa_{3}+\kappa_{4}\right) H_{6}(z)+\frac{1}{1296} \kappa_{3}^{3} H_{8}(z)+O\left(n^{-2}\right)\right] .
\end{aligned}
$$

This expansion assumes, as is the case for $R_{\mathrm{LR}}$, that $\kappa_{2}=1+O\left(n^{-1}\right)$. (Expansions for statistics in which $\kappa_{2}=1+O\left(n^{-1 / 2}\right)$, e.g., $R_{\mathrm{W} 4}$, are more complicated.) To compute the cumulants of $R_{\mathrm{LR}}$, introduce, similarly to $\rho$ and $\gamma$ defined as before, the (dimensionless) quantity

$$
\xi=\frac{V_{5}}{24\left(-V_{2}\right)^{5 / 2}}=\frac{\mathbb{E}_{s}\left[\frac{s^{4}}{b^{4}}\right]-5 \mathbb{E}_{s}\left[\frac{s^{3}}{b^{3}}\right]+10 \mathbb{E}_{s}\left[\frac{s^{2}}{b^{2}}\right]-10 \mathbb{E}_{s}\left[\frac{s}{b}\right]+4}{\left(\mathbb{E}_{s}\left[\frac{s}{b}\right]-1\right)^{5 / 2}} .
$$

In terms of these parameters, and to an accuracy of $O\left(n^{-2}\right)$, we obtain the following expressions for the first five cumulants of $R_{\mathrm{LR}}$ :

$$
\begin{aligned}
& \kappa_{1}=-\frac{\gamma}{6} n^{-1 / 2}+\left(\frac{\gamma}{16}-\frac{\gamma^{3}}{12}+\frac{5 \gamma \rho}{16}-\frac{11 \xi}{40}\right) n^{-3 / 2} \\
& \kappa_{2}=1+\frac{18 \rho-13 \gamma^{2}}{36 n}, \quad \kappa_{3}=\left(\frac{11 \gamma \rho}{4}-\frac{251 \gamma^{3}}{216}-\frac{9 \xi}{5}\right) n^{-3 / 2},
\end{aligned}
$$

with $\kappa_{4}=\kappa_{5}=0$. Substitution of these cumulants into (22) gives:

$$
\begin{aligned}
\operatorname{Pr}\left(R_{\mathrm{LR}} \leq z\right)= & \Phi(z)-\phi(z)\left\{n^{-1 / 2}\left(-\frac{\gamma}{6}\right)+n^{-1}\left(\frac{1}{12}\left(3 \rho-2 \gamma^{2}\right) z\right)\right. \\
& \left.+n^{-3 / 2}\left(\frac{\gamma}{16}-\frac{\gamma^{3}}{12}+\frac{5 \gamma \rho}{16}-\frac{11 \xi}{40}+\left[\frac{5 \gamma \rho}{12}-\frac{71 \gamma^{3}}{432}-\frac{3 \xi}{10}\right] H_{2}(z)\right)+O\left(n^{-2}\right)\right\} .
\end{aligned}
$$


For the model settings as in the illustrative example of section $4, \xi \approx 5.0$. The $O\left(n^{-2}\right)$ prediction agrees with the simulations better than the $O\left(n^{-3 / 2}\right)$ prediction in terms of the mean, standard deviation, skewness, kurtosis, and $\chi^{2}$ test values listed in Tables 8-12.

\section{Assessment of Normality for the Statistics in Table 2}

The information in Tables 8-11 gauges how closely the mean, standard deviation, skewness, and kurtosis of the statistics in Table 2 agree with the corresponding values predicted by $\mathcal{N}(0,1)$ and $O\left(n^{-3 / 2}\right)$ models. The simulated values are constructed from $m=10^{9}$ replications. If $r_{1}, \ldots, r_{m}$ denote the $m$ simulated values of a particular statistic with empirical mean $\bar{r}=\sum r_{i} / m$, define the empirical $k$-th central moment as $\mu_{k}=\sum\left(r_{i}-\bar{r}\right)^{k} / m$. The "simulated value $(S V)$ " and "simulation uncertainty $(S U)$ " quantities are, respectively, the appropriate empirical moment estimate, and the standard deviation of the estimate, determined as follows (see [9] for details).

- Table 8 for the mean: $S V=\bar{r}$, and $S U=\frac{1}{\sqrt{m}}=3.2 \times 10^{-5}$.

- Table 9 for the standard deviation minus 1 ( $S U$ assumes the sample is drawn from a $\mathcal{N}(0,1)$ ): $S V=\sqrt{\mu_{2}}-1$, and $S U=1 / \sqrt{2(m-1)}=2.2 \times 10^{-5}$.

- Table 10 for the skewness coefficient ( $S U$ assumes the sample is drawn from a $\mathcal{N}(0,1)$ ):

$$
S V=\frac{\mu_{3}}{\mu_{2}^{3 / 2}}, \quad S U=\sqrt{\frac{6 m(m-1)}{(m-2)(m+1)(m+3)}}=7.7 \times 10^{-5} .
$$

- Table 11 for the kurtosis coefficient ( $S U$ assumes the sample is drawn from a $\mathcal{N}(0,1)$ ):

$$
S V=\frac{\mu_{4}}{\mu_{2}^{2}}-3, \quad S U=\sqrt{\frac{24 m(m-1)^{2}}{(m-3)(m-2)(3+m)(5+m)}}=1.5 \times 10^{-4} .
$$

Table 12 gives the results of a chi-square $\left(\chi^{2}\right)$ goodness-of-fit test. Using a bin width of 0.1 (and utilizing all bins with 25 or more predicted counts), the proportions of the respective $R$ statistic values falling in each bin over the $m=10^{9}$ replications are converted to a single $\chi^{2}$-value comparing the observed proportions with predicted probabilities for a $\mathcal{N}(0,1)$, and second and third order Edgeworth approximations. Each $\chi^{2}$-value is then converted to a corresponding $p$ value for the test by calculating the survival probability at the value under a $\chi^{2}$ distribution with the indicated degrees of freedom. 
Table 8: Comparison of the first cumulant from Table 2 with the simulation-based empirical estimate constructed from $10^{9}$ replications. Predictions that differ from simulated values by more than twice the simulation uncertainty appear in bold face. The corresponding $\mathcal{N}(0,1)$ value is 0 .

\begin{tabular}{|c|c|c|c|c|}
\hline & $n$ & $\begin{array}{c}O\left(n^{-3 / 2}\right) \\
\text { Prediction }\end{array}$ & $\begin{array}{l}\text { Simulated } \\
\text { Value }\end{array}$ & $\begin{array}{l}\text { Simulation } \\
\text { Uncertainty }\end{array}$ \\
\hline$R_{\mathrm{W}}$ & $\begin{array}{r}200 \\
1000 \\
5000 \\
25000\end{array}$ & $\begin{array}{l}\mathbf{0} \\
0 \\
0 \\
0\end{array}$ & $\begin{aligned}-9.0 & \times 10^{-5} \\
-0.9 & \times 10^{-5} \\
-1.6 & \times 10^{-5} \\
4.8 & \times 10^{-5}\end{aligned}$ & $3.2 \times 10^{-5}$ \\
\hline$R_{\mathrm{W} 2}$ & $\begin{array}{r}200 \\
1000 \\
5000 \\
25000\end{array}$ & $\begin{array}{r}-\mathbf{3 9 . 2 2 2} \times \mathbf{1 0}^{-\mathbf{3}} \\
\mathbf{- 1 7 . 5 4 1} \times \mathbf{1 0}^{-\mathbf{3}} \\
-7.844 \times 10^{-3} \\
-3.508 \times 10^{-3}\end{array}$ & $\begin{aligned}-40.724 & \times 10^{-3} \\
-17.670 & \times 10^{-3} \\
-7.872 & \times 10^{-3} \\
-3.461 & \times 10^{-3}\end{aligned}$ & $0.032 \times 10^{-3}$ \\
\hline$R_{\mathrm{W} 3}$ & $\begin{array}{r}200 \\
1000 \\
5000 \\
25000\end{array}$ & $\begin{array}{r}-\mathbf{3 9 . 2 2 2} \times \mathbf{1 0}^{-3} \\
-\mathbf{1 7 . 5 4 1} \times \mathbf{1 0}^{-\mathbf{3}} \\
-7.844 \times 10^{-3} \\
-3.508 \times 10^{-3}\end{array}$ & $\begin{array}{r}-40.817 \times 10^{-3} \\
-17.678 \times 10^{-3} \\
-7.872 \times 10^{-3} \\
-3.461 \times 10^{-3}\end{array}$ & $0.032 \times 10^{-3}$ \\
\hline$R_{\mathrm{W} 4}$ & $\begin{array}{r}200 \\
1000 \\
5000 \\
25000\end{array}$ & $\begin{aligned}-50.617 & \times 10^{-3} \\
-19.820 & \times 10^{-3} \\
-8.300 & \times 10^{-3} \\
-3.599 & \times 10^{-3}\end{aligned}$ & $\begin{array}{r}-48.508 \times 10^{-3} \\
-19.106 \times 10^{-3} \\
-8.152 \times 10^{-3} \\
-3.516 \times 10^{-3}\end{array}$ & $0.032 \times 10^{-3}$ \\
\hline$R_{\mathrm{LR}}$ & $\begin{array}{r}200 \\
1000 \\
5000 \\
25000\end{array}$ & $\begin{array}{r}-\mathbf{1 3 . 0 7 4} \times \mathbf{1 0}^{-\mathbf{3}} \\
-5.847 \times 10^{-3} \\
-2.615 \times 10^{-3} \\
-1.169 \times 10^{-3}\end{array}$ & $\begin{array}{r}-13.199 \times 10^{-3} \\
-5.860 \times 10^{-3} \\
-2.631 \times 10^{-3} \\
-1.121 \times 10^{-3}\end{array}$ & $0.032 \times 10^{-3}$ \\
\hline$R_{\mathrm{S}}$ & $\begin{array}{r}200 \\
1000 \\
5000 \\
25000\end{array}$ & $\begin{array}{l}0 \\
0 \\
0 \\
0\end{array}$ & $\begin{aligned} 2.7 \times 10^{-5} \\
-1.6 \times 10^{-5} \\
1.3 \times 10^{-5} \\
0.1 \times 10^{-5}\end{aligned}$ & $3.2 \times 10^{-5}$ \\
\hline
\end{tabular}


Table 9: Comparison of the standard deviation minus one, constructed from the cumulants in Table 2, with the simulation-based empirical estimate over $10^{9}$ replications. Predictions that differ from simulated values by more than twice the simulation uncertainty appear in bold face. The corresponding $\mathcal{N}(0,1)$ value is 0 .

\begin{tabular}{|c|c|c|c|c|}
\hline & $n$ & $\begin{array}{c}O\left(n^{-3 / 2}\right) \\
\text { Prediction }\end{array}$ & $\begin{array}{l}\text { Simulated } \\
\text { Value }\end{array}$ & $\begin{array}{l}\text { Simulation } \\
\text { Uncertainty }\end{array}$ \\
\hline$R_{\mathrm{W}}$ & $\begin{array}{r}200 \\
1000 \\
5000 \\
25000\end{array}$ & 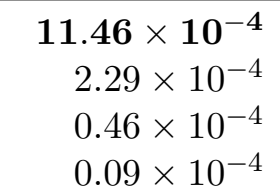 & $\begin{aligned} 11.97 & \times 10^{-4} \\
2.60 & \times 10^{-4} \\
0.76 & \times 10^{-4} \\
-0.30 & \times 10^{-4}\end{aligned}$ & $0.22 \times 10^{-4}$ \\
\hline$R_{\mathrm{W} 2}$ & $\begin{array}{r}200 \\
1000 \\
5000 \\
25000\end{array}$ & $\begin{aligned} \mathbf{1 6 1 . 9 3} & \times \mathbf{1 0}^{-4} \\
\mathbf{3 2 . 5 9} & \times \mathbf{1 0}^{-4} \\
6.53 & \times 10^{-4} \\
1.31 & \times 10^{-4}\end{aligned}$ & $\begin{array}{r}174.00 \times 10^{-4} \\
33.31 \times 10^{-4} \\
6.86 \times 10^{-4} \\
0.92 \times 10^{-4}\end{array}$ & $0.22 \times 10^{-4}$ \\
\hline$R_{\mathrm{W} 3}$ & $\begin{array}{r}200 \\
1000 \\
5000 \\
25000\end{array}$ & $\begin{aligned} \mathbf{1 7 8 . 1 7} & \times \mathbf{1 0}^{-4} \\
\mathbf{3 5 . 8 9} & \times \mathbf{1 0}^{-4} \\
7.19 & \times 10^{-4} \\
1.44 & \times 10^{-4}\end{aligned}$ & $\begin{aligned} 191.06 & \times 10^{-4} \\
36.63 & \times 10^{-4} \\
7.52 & \times 10^{-4} \\
1.05 & \times 10^{-4}\end{aligned}$ & $0.22 \times 10^{-4}$ \\
\hline$R_{\mathrm{W} 4}$ & $\begin{array}{r}200 \\
1000 \\
5000 \\
25000\end{array}$ & $\begin{aligned} 441.74 & \times 10^{-4} \\
153.76 & \times \mathbf{1 0}^{-4} \\
59.72 & \times 10^{-4} \\
24.88 & \times 10^{-4}\end{aligned}$ & $\begin{aligned} 491.83 & \times 10^{-4} \\
157.40 & \times 10^{-4} \\
60.30 & \times 10^{-4} \\
24.51 & \times 10^{-4}\end{aligned}$ & $0.22 \times 10^{-4}$ \\
\hline$R_{\mathrm{LR}}$ & $\begin{array}{r}200 \\
1000 \\
5000 \\
25000\end{array}$ & $\begin{aligned} 22.48 & \times 10^{-4} \\
4.50 & \times 10^{-4} \\
0.90 & \times 10^{-4} \\
0.18 & \times 10^{-4}\end{aligned}$ & $\begin{aligned} 22.86 & \times 10^{-4} \\
4.79 & \times 10^{-4} \\
1.21 & \times 10^{-4} \\
-0.21 & \times 10^{-4}\end{aligned}$ & $0.22 \times 10^{-4}$ \\
\hline$R_{\mathrm{S}}$ & $\begin{array}{r}200 \\
1000 \\
5000 \\
25000\end{array}$ & $\begin{array}{l}0 \\
0 \\
0 \\
\mathbf{0}\end{array}$ & $\begin{aligned}-1.5 & \times 10^{-5} \\
0.1 & \times 10^{-5} \\
3.8 & \times 10^{-5} \\
4.7 & \times 10^{-5}\end{aligned}$ & $2.2 \times 10^{-5}$ \\
\hline
\end{tabular}


Table 10: Comparison of the skewness coefficient, constructed from the cumulants in Table 2, with the simulation-based empirical estimate over $10^{9}$ replications. Predictions that differ from simulated values by more than twice the simulation uncertainty appear in bold face. The corresponding $\mathcal{N}(0,1)$ value is 0 .

\begin{tabular}{|c|c|c|c|c|}
\hline & $n$ & $\begin{array}{c}O\left(n^{-3 / 2}\right) \\
\text { Prediction }\end{array}$ & $\begin{array}{l}\text { Simulated } \\
\text { Value }\end{array}$ & $\begin{array}{l}\text { Simulation } \\
\text { Uncertainty }\end{array}$ \\
\hline \multirow{4}{*}{$R_{\mathrm{W}}$} & 200 & 0.07817 & 0.07747 & \multirow{4}{*}{0.00008} \\
\hline & 1000 & 0.03506 & 0.03513 & \\
\hline & 5000 & 0.01569 & 0.01562 & \\
\hline & 25000 & 0.00702 & 0.00701 & \\
\hline \multirow{4}{*}{$R_{\mathrm{W} 2}$} & 200 & -0.14951 & -0.17543 & \multirow{4}{*}{0.00008} \\
\hline & 1000 & -0.06948 & -0.07150 & \\
\hline & 5000 & -0.03132 & -0.03157 & \\
\hline & 25000 & -0.01402 & -0.01405 & \\
\hline \multirow{4}{*}{$R_{\mathrm{W} 3}$} & 200 & -0.14879 & -0.17563 & \multirow{4}{*}{0.00008} \\
\hline & 1000 & -0.06941 & -0.07152 & \\
\hline & 5000 & -0.03131 & -0.03157 & \\
\hline & 25000 & -0.01402 & -0.01405 & \\
\hline \multirow{4}{*}{$R_{\mathrm{W} 4}$} & 200 & -0.17965 & -0.21724 & \multirow{4}{*}{0.00008} \\
\hline & 1000 & -0.07612 & -0.07883 & \\
\hline & 5000 & -0.03269 & -0.03300 & \\
\hline & 25000 & -0.01431 & -0.01434 & \\
\hline \multirow{4}{*}{$R_{\mathrm{LR}}$} & 200 & 0 & -0.00084 & \multirow{4}{*}{0.00008} \\
\hline & 1000 & 0 & 0.00006 & \\
\hline & 5000 & 0 & -0.00007 & \\
\hline & 25000 & 0 & -0.00001 & \\
\hline \multirow{4}{*}{$R_{\mathrm{S}}$} & 200 & 0.07844 & 0.07853 & \multirow{4}{*}{0.00008} \\
\hline & 1000 & 0.03508 & 0.03501 & \\
\hline & 5000 & 0.01569 & 0.01567 & \\
\hline & 25000 & 0.00702 & 0.00693 & \\
\hline
\end{tabular}


Table 11: Comparison of the excess kurtosis coefficient, constructed from the cumulants in Table 2, with the simulation-based empirical estimate over $10^{9}$ replications. Predictions that differ from simulated values by more than twice the simulation uncertainty appear in bold face. The corresponding $\mathcal{N}(0,1)$ value is 0 .

\begin{tabular}{|c|c|c|c|c|}
\hline & $n$ & $\begin{array}{c}O\left(n^{-3 / 2}\right) \\
\text { Prediction }\end{array}$ & $\begin{array}{l}\text { Simulated } \\
\text { value }\end{array}$ & $\begin{array}{l}\text { Simulation } \\
\text { Uncertainty }\end{array}$ \\
\hline$R_{\mathrm{W}}$ & $\begin{array}{r}200 \\
1000 \\
5000 \\
25000\end{array}$ & $\begin{array}{r}-\mathbf{1 5 . 5} \times \mathbf{1 0}^{-\mathbf{4}} \\
-3.1 \times 10^{-4} \\
-0.6 \times 10^{-4} \\
-0.1 \times 10^{-4}\end{array}$ & $\begin{aligned}-10.6 & \times 10^{-4} \\
-3.6 & \times 10^{-4} \\
-2.7 & \times 10^{-4} \\
2.6 & \times 10^{-4}\end{aligned}$ & $1.5 \times 10^{-4}$ \\
\hline$R_{\mathrm{W} 2}$ & $\begin{array}{r}200 \\
1000 \\
5000 \\
25000\end{array}$ & $\begin{array}{r}\mathbf{1 2 6 . 1 0} \times \mathbf{1 0}^{-3} \\
\mathbf{2 6 . 5 4} \times \mathbf{1 0}^{-\mathbf{3}} \\
5.36 \times 10^{-3} \\
1.08 \times 10^{-3}\end{array}$ & $\begin{array}{r}163.24 \times 10^{-3} \\
27.76 \times 10^{-3} \\
5.24 \times 10^{-3} \\
1.35 \times 10^{-3}\end{array}$ & $0.15 \times 10^{-3}$ \\
\hline$R_{\mathrm{W} 3}$ & $\begin{array}{r}200 \\
1000 \\
5000 \\
25000\end{array}$ & $\begin{array}{r}\mathbf{1 2 5 . 2 9} \times \mathbf{1 0}^{-\mathbf{3}} \\
\mathbf{2 6 . 5 1} \times \mathbf{1 0}^{-\mathbf{3}} \\
5.36 \times 10^{-3} \\
1.08 \times 10^{-3}\end{array}$ & $\begin{array}{r}163.60 \times 10^{-3} \\
27.77 \times 10^{-3} \\
5.24 \times 10^{-3} \\
1.35 \times 10^{-3}\end{array}$ & $0.15 \times 10^{-3}$ \\
\hline$R_{\mathrm{W} 4}$ & $\begin{array}{r}200 \\
1000 \\
5000 \\
25000\end{array}$ & $\begin{array}{r}\mathbf{1 1 3 . 1 1} \times \mathbf{1 0}^{-\mathbf{3}} \\
\mathbf{2 5 . 3 0} \times \mathbf{1 0}^{-\mathbf{3}} \\
5.25 \times 10^{-3} \\
1.07 \times 10^{-3}\end{array}$ & $\begin{array}{r}207.78 \times 10^{-3} \\
30.56 \times 10^{-3} \\
5.47 \times 10^{-3} \\
1.37 \times 10^{-3}\end{array}$ & $0.15 \times 10^{-3}$ \\
\hline$R_{\mathrm{LR}}$ & $\begin{array}{r}200 \\
1000 \\
5000 \\
25000\end{array}$ & $\begin{array}{l}\mathbf{0} \\
0 \\
0 \\
0\end{array}$ & $\begin{array}{r}3.7 \times 10^{-4} \\
-0.6 \times 10^{-4} \\
-2.0 \times 10^{-4} \\
2.7 \times 10^{-4}\end{array}$ & $1.5 \times 10^{-4}$ \\
\hline$R_{\mathrm{S}}$ & $\begin{array}{r}200 \\
1000 \\
5000 \\
25000\end{array}$ & $\begin{aligned}-15.5 & \times 10^{-4} \\
-3.1 & \times 10^{-4} \\
-0.6 & \times 10^{-4} \\
-0.1 & \times 10^{-4}\end{aligned}$ & $\begin{aligned}-14.6 & \times 10^{-4} \\
-2.7 & \times 10^{-4} \\
0.7 & \times 10^{-4} \\
2.3 & \times 10^{-4}\end{aligned}$ & $1.5 \times 10^{-4}$ \\
\hline
\end{tabular}


Table 12: $\chi^{2}$ goodness-of-fit test for the statistics in Table 2 with $\mathcal{N}(0,1)$, and second and third order Edgeworth predictions. For each $n$, the test is constructed from $10^{9}$ replications using a bin width of 0.1 and all bins with 25 or more predicted counts. Entries are formated as $\{a / b$, $c\}$, where $a$ is the $\chi^{2}$ statistic value, $b$ is the number of degrees of freedom (i.e., the number of bins used), and $c$ is the corresponding $p$-value.

\begin{tabular}{|c|c|c|c|c|}
\hline & $n$ & $\begin{array}{c}\mathcal{N}(0,1) \\
\text { prediction }\end{array}$ & $\begin{array}{c}O\left(n^{-1}\right) \\
\text { prediction }\end{array}$ & $\begin{array}{c}O\left(n^{-3 / 2}\right) \\
\text { prediction }\end{array}$ \\
\hline \multirow{4}{*}{$R_{\mathrm{W}}$} & 200 & 1012631/107, 0.00 & $12920 / 100, \quad 0.00$ & $358.7 / 107,0.00$ \\
\hline & 1000 & $205959 / 107, \quad 0.00$ & $534.4 / 106,0.00$ & $124.6 / 106, \quad 0.10$ \\
\hline & 5000 & $40702 / 107, \quad 0.00$ & $110.2 / 107, \quad 0.40$ & $90.29 / 107, \quad 0.88$ \\
\hline & 25000 & $8254 / 107, \quad 0.00$ & $81.34 / 107, \quad 0.97$ & $82.37 / 107,0.96$ \\
\hline \multirow{4}{*}{$R_{\mathrm{W} 2}$} & 200 & $11789846 / 107, \quad 0.00$ & $3710820 / 92, \quad 0.00$ & $402208 / 116, \quad 0.00$ \\
\hline & 1000 & 1266791/107, 0.00 & $105137 / 101,0.00$ & $4104 / 110, \quad 0.00$ \\
\hline & 5000 & $231468 / 107,0.00$ & $2967 / 106, \quad 0.00$ & $169.5 / 107, \quad 0.00$ \\
\hline & 25000 & $44997 / 107, \quad 0.00$ & $196.9 / 107,0.00$ & $78.53 / 107, \quad 0.98$ \\
\hline \multirow{4}{*}{$R_{\mathrm{W} 3}$} & 200 & $12178306 / 107,0.00$ & $3961404 / 92, \quad 0.00$ & $426251 / 116, \quad 0.00$ \\
\hline & 1000 & $1275312 / 107, \quad 0.00$ & $110857 / 101, \quad 0.00$ & $4292 / 110, \quad 0.00$ \\
\hline & 5000 & $231797 / 107, \quad 0.00$ & $3169 / 106, \quad 0.00$ & $171.2 / 107, \quad 0.00$ \\
\hline & 25000 & $45006 / 107, \quad 0.00$ & $201.8 / 107,0.00$ & $77.90 / 107,0.98$ \\
\hline \multirow{4}{*}{$R_{\mathrm{W} 4}$} & 200 & $31650126 / 107, \quad 0.00$ & $5860538 / 95, \quad 0.00$ & $1352453 / 116,0.00$ \\
\hline & 1000 & $2166332 / 107, \quad 0.00$ & $116183 / 103, \quad 0.00$ & $8056 / 110, \quad 0.00$ \\
\hline & 5000 & $333907 / 107,0.00$ & 4365/106, 0.00 & $212.1 / 108,0.00$ \\
\hline & 25000 & $59517 / 107, \quad 0.00$ & $248.1 / 107, \quad 0.00$ & $87.67 / 107, \quad 0.92$ \\
\hline \multirow{4}{*}{$R_{\mathrm{LR}}$} & 200 & $185597 / 107, \quad 0.00$ & $11493 / 107,0.00$ & $292.0 / 107, \quad 0.00$ \\
\hline & 1000 & $34917 / 107, \quad 0.00$ & $616.9 / 107, \quad 0.00$ & $126.5 / 107, \quad 0.10$ \\
\hline & 5000 & $7068 / 107, \quad 0.00$ & $142.5 / 107,0.01$ & $113.8 / 107,0.31$ \\
\hline & 25000 & $1339 / 107,0.00$ & $80.78 / 107, \quad 0.97$ & $82.95 / 107,0.96$ \\
\hline \multirow{4}{*}{$R_{\mathrm{S}}$} & 200 & $1030516 / 107,0.00$ & $8697 / 100,0.00$ & $299.3 / 106, \quad 0.00$ \\
\hline & 1000 & $204171 / 107, \quad 0.00$ & $314.2 / 106, \quad 0.00$ & $116.5 / 106, \quad 0.23$ \\
\hline & 5000 & $40879 / 107,0.00$ & $95.88 / 107, \quad 0.77$ & $92.46 / 107, \quad 0.84$ \\
\hline & 25000 & $8100 / 107, \quad 0.00$ & $107.2 / 107,0.48$ & $108.5 / 107,0.44$ \\
\hline
\end{tabular}

